TRANSACTIONS OF THE

AMERICAN MATHEMATICAL SOCIETY

Volume 359, Number 10, October 2007, Pages 5035-5056

S 0002-9947(07)04193-1

Article electronically published on April 16, 2007

\title{
LIMIT WEIERSTRASS POINTS ON NODAL REDUCIBLE CURVES
}

\author{
EDUARDO ESTEVES AND PARHAM SALEHYAN
}

\begin{abstract}
In the 1980s D. Eisenbud and J. Harris posed the following question: "What are the limits of Weierstrass points in families of curves degenerating to stable curves not of compact type?" In the present article, we give a partial answer to this question. We consider the case where the limit curve has components intersecting at points in general position and where the degeneration occurs along a general direction. For this case we compute the limits of Weierstrass points of any order. However, for the usual Weierstrass points, of order one, we need to suppose that all of the components of the limit curve intersect each other.
\end{abstract}

\section{INTRODUCTION}

Limits of ramification points and linear systems were studied by Eisenbud and Harris in the 1980's, when they developed the theory of limit linear series for curves of compact type; see 2]. Many important applications of their theory were found; a survey is given in [2] as well.

As far as Weierstrass points are concerned, in 22 Eisenbud and Harris developed a theory to identify limits of linear systems and ramification points as smooth curves degenerate to curves of compact type, and in [3] they applied their theory to the study of limits of Weierstrass points.

The results in [4] allow us to identify limits of linear systems and ramification points as smooth curves degenerate to reducible nodal curves of any type. In the present article, we show how to apply these results to the study of limits of canonical systems and Weierstrass points. We also deal with higher Weierstrass points.

Computing limits of canonical systems can be difficult without further hypotheses. In the present work we compute them when the components of the limit curve intersect in general position and the degeneration occurs along a general direction. When we deal with Weierstrass points of order one, we also suppose that all of the components of the limit curve intersect each other.

We will now proceed to explain briefly the main result of the article, our Theorem 6. Let $C$ be a projective, connected and nodal curve of arithmetic genus $g \geq 2$ defined over an algebraically closed field $k$ of characteristic zero, and $C_{1}, \ldots, C_{t}$ its irreducible components. Let $g_{i}$ be the arithmetic genus of $C_{i}$ for each $i$. To avoid known or special cases, we will always assume $t>1$ and $g_{i}>0$ for each $i$.

Received by the editors April 4, 2005 and, in revised form, September 19, 2005.

2000 Mathematics Subject Classification. Primary 14H10, 14H55.

The first author was supported by CNPq, Proc. 300004/95-8.

The second author was supported by CNPq, Proc. 142643/98-0 and 150258/03-8. 
Consider now a one-parameter infinitesimal smoothing of $C$. More precisely, set $S:=\operatorname{Spec}(k[[u]])$ and let $f: \mathcal{X} \rightarrow S$ be a flat, projective map whose special fiber is $C$ and whose generic fiber is smooth. Assume the total space $\mathcal{X}$ is regular, which is the case for a smoothing along a general direction in the versal deformation space of $C$. Then the components $C_{i}$ are Cartier divisors on $\mathcal{X}$. For each $i=1, \ldots, t$, let $N_{i}:=\left.\mathcal{O}_{\mathcal{X}}\left(C_{i}\right)\right|_{C}$. We say that the collection $N_{1}, \ldots, N_{t}$ is (an ordered basis of) an enriched structure on $C$; cf. [7].

Necessary and sufficient conditions for a collection of invertible sheaves $N_{1}, \ldots, N_{t}$ on $C$ to form an enriched structure were found in [7, Prop. 3.16; see also 6], Thm. 6.10, p. 297. For all distinct $i, j \in\{1, \ldots, t\}$, let $\Delta_{i, j}$ denote the reduced Weil divisor on $C$ with support $C_{i} \cap C_{j}$, and put $\delta_{i, j}:=\operatorname{deg} \Delta_{i, j}$. For each $i=1, \ldots, t$, let $\widehat{C}_{i}:=\overline{C-C_{i}}$ and $\Delta_{i}:=\sum_{j \neq i} \Delta_{i, j}$. The conditions are:

$$
N_{1} \otimes \cdots \otimes N_{t} \cong \mathcal{O}_{C} \text { and }\left.N_{i}\right|_{\widehat{C}_{i}} \cong \mathcal{O}_{\widehat{C}_{i}}\left(\Delta_{i}\right) \text { for every } i=1, \ldots, t .
$$

Let $K$ be the canonical sheaf on $C$ and $\beta$ any positive integer. Consider the $\beta$-canonical system $H^{0}\left(C, K^{\otimes \beta}\right)$. This system is usually degenerate on each component of $C$; see [1] for its study.

However, assume that the components of $C$ intersect at points in general position. (A precise definition of "general position" is given at the beginning of Section 4.) In addition, if $\beta=1$, assume that $\delta_{i, j} \neq 0$ for all $i$ and $j$; if $\beta>1$, assume that $g_{i} \geq 2$ for every $i$. Then we show that, for each $\ell=1, \ldots, t$, there is a $t$-tuple $\underline{n}^{(\ell)}=\left(n_{1}^{(\ell)}, \ldots, n_{t}^{(\ell)}\right)$ of integers, with $n_{\ell}^{(\ell)}=0$, such that, for a general collection of invertible sheaves $N_{1}, \ldots, N_{t}$ on $C$ forming an enriched structure, the complete linear system of sections of the modified $\beta$-canonical sheaf

$$
L^{(\ell)}:=K^{\otimes \beta} \otimes N_{1}^{\otimes n_{1}^{(\ell)}} \otimes \cdots \otimes N_{t}^{\otimes n_{t}^{(\ell)}}
$$

has finitely many base points, is nondegenerate on $C_{\ell}$ and has rank $h^{0}\left(C, K^{\otimes \beta}\right)-1$. This is an immediate consequence of our Proposition 5

The $t$-tuple $\underline{n}^{(\ell)}$ is given by Lemma 4 , for the special $t$-tuple $\underline{e}^{(\ell)}$ defined at the beginning of Section 6 . The proof of the lemma yields an algorithm for computing $\underline{n}^{(\ell)}$.

Finally, let $f: \mathcal{X} \rightarrow S$ be a smoothing of $C$ along a general direction. Consider on the generic fiber of $f$ the Weierstrass (Cartier) divisor of the $\beta$-canonical system, and let $W$ be the fundamental cycle on $C$ associated to the subscheme that is the limit of this divisor. We call $W$ the limit Weierstrass divisor of order $\beta$.

Since the smoothing $f$ is taken along a general direction, the resulting enriched structure, $N_{1}, \ldots, N_{t}$, is also general. For each $\ell=1, \ldots, t$, let $W_{\ell}$ be the ramification Weil divisor of the space of sections of $\left.L^{(\ell)}\right|_{C_{\ell}}$ generated by $H^{0}\left(C, L^{(\ell)}\right)$, with $L^{(\ell)}$ as above. Then we show that, as a Weil divisor,

$$
W=\sum_{\ell=1}^{t} W_{\ell}+\sum_{i<j} \kappa_{\beta}\left(\kappa_{\beta}-1-n_{j}^{(i)}-n_{i}^{(j)}\right) \Delta_{i, j},
$$

where $\kappa_{\beta}:=h^{0}\left(C, K^{\otimes \beta}\right)$. (Notice that, under our hypotheses, $\kappa_{\beta}=g$ if $\beta=1$ and $\kappa_{\beta}=(2 \beta-1)(g-1)$ if $\beta>1$.) This is our Theorem 6 .

Proposition 5 implies that $H^{0}\left(C, L^{(\ell)}\right)$ is the limit linear system associated to $C_{\ell}$; see 4, p. 26. Thus the expression for $W$ is a direct consequence of 4], Thm. 7, p. 30. Therefore, the bulk of the article consists of showing Proposition 5. 
How important are the assumptions we make? First, it is necessary to assume that the components intersect at points in general position. In [3], Section 4, p. 508, for a two-component curve $C$ with a single node, it is shown how the limit Weierstrass points on $C$ may vary wildly when the node is a Weierstrass point of one of the components. Second, for $\beta=1$ our approach does not apply to curves of compact type, as a comparison with the formula prescribed in [3], Thm. 3.1, p. 507 shows; see also our Example after Theorem 6. Therefore, a condition like " $\delta_{i, j} \neq 0$ for all $i$ and $j "$ is necessary. Third, it is necessary to assume that the smoothing occurs along a general direction, as our Example after Proposition 5 shows.

In [5] and [6] limits of Weierstrass points on two-component curves are described in detail. In [5] smoothings along general directions (for which the total space of the smoothing is regular) were studied, whereas in [6] all smoothings were considered. The present article is thus a generalization of [5]. It might be possible, and interesting, to pursue our study further, and consider all smoothings of a given curve. However, a formula for $W$ generalizing both that in [3] and the one here would be necessary, as after semistable reduction the condition " $\delta_{i, j} \neq 0$ for all $i$ and $j$ " will not hold.

So this article can only be viewed as a small step, after many years, in understanding limits of Weierstrass points in general. We do not know whether applications, beyond what Eisenbud and Harris did in [2], are possible. Our feeling is that we need first to know more about those limits.

In Section 2 we set up the notation. In Section 3, we consider an invertible sheaf $\widetilde{L}$ on the partial normalization $\widetilde{C}$ of a curve $C$ along a set of nodes, and compute $h^{0}(C, L)$ for the general invertible sheaf $L$ on $C$ pulling back to $\widetilde{L}$. In Section 4 , we apply this computation to show that, under general conditions, modified $\beta$ canonical sheaves also have low first cohomology. In Section 5 we show a purely numerical result, the existence and uniqueness of a $t$-tuple of integers $\left(n_{1}, \ldots, n_{t}\right)$ satisfying certain numerical inequalities. In Section 6 we apply the results of Section 4 and Section 5 to derive our main result.

\section{Smoothings}

Let $C$ be a projective, connected and nodal curve defined over an algebraically closed field $k$ of characteristic zero, and $C_{1}, \ldots, C_{t}$ its irreducible components. Let $\beta$ be a positive integer. Denote by $K^{\beta}$ the $\beta$-canonical sheaf of $C$, and by $g$ the arithmetic genus.

Recall that the degree of a Weil divisor $\sum n_{p} p$ on $C$ is the sum $\sum n_{p}$. All of the divisors in this article are assumed to be Weil, unless explicitly said to be Cartier. A Weil divisor on $C$ can and will be considered Cartier on the subcurves of $C$ containing the support of the divisor in their nonsingular loci.

Assume $t>1$. For all distinct $i, j \in\{1, \ldots, t\}$ let $\Delta_{i, j}$ denote the reduced Weil divisor on $C$ whose support is $C_{i} \cap C_{j}$, and put $\delta_{i, j}:=\operatorname{deg} \Delta_{i, j}$. Let

$$
\delta:=\sum_{i<j} \delta_{i, j}
$$

For each $i=1, \ldots, t$, let $\Delta_{i}:=\sum_{j \neq i} \Delta_{i, j}$ and $\delta_{i}:=\operatorname{deg} \Delta_{i}$.

For each nonempty subset $I \subseteq\{1, \ldots, t\}$, let

$$
C_{I}:=\bigcup_{i \in I} C_{i}
$$


let $K_{I}^{\beta}$ denote the $\beta$-canonical sheaf of $C_{I}$, and $g_{I}$ the arithmetic genus, given by $g_{I}:=h^{0}\left(C_{I}, K_{I}\right)$. The relation between the $\beta$-canonical sheaves of $C$ and $C_{I}$ follows from Lemma 1.12 in [1], p. 61:

$$
\left.K^{\beta}\right|_{C_{I}} \cong K_{I}^{\beta}\left(\sum_{i \in I} \sum_{j \notin I} \Delta_{i, j}\right) .
$$

For simplicity, when appearing as an index, the subset $\{i\}$ will be replaced by $i$. We say that $I$ is connected if $C_{I}$ is connected.

If $I$ is connected, since $C$ is nodal, Formula 1.6 in [1], p. 57, yields:

$$
g_{I}=\sum_{i \in I} g_{i}+\sum_{\substack{i, j \in I \\ i<j}} \delta_{i, j}-\# I+1 \geq \sum_{i \in I} g_{i} .
$$

In particular, since $C$ is assumed connected, $\delta \geq t-1$ and

$$
g=g_{1}+\cdots+g_{t}+\delta-t+1 .
$$

Assume $g_{i}>0$ for each $i=1, \ldots, t$.

Let $S$ be the spectrum of a discrete valuation ring with residue field $k$. Let $s$ (resp. $\eta$ ) denote the special (resp. generic) point of $S$. A smoothing of $C$ is a flat, projective map $f: \mathcal{X} \rightarrow S$ whose generic fiber $\mathcal{X}(\eta)$ is smooth, and whose special fiber $\mathcal{X}(s)$ is isomorphic to $C$. If $\mathcal{X}$ is regular, the smoothing is called regular.

Let $f: \mathcal{X} \rightarrow S$ be a regular smoothing of $C$. Since $\mathcal{X}$ is regular, $C_{1}, \ldots, C_{t}$ are Cartier divisors on $\mathcal{X}$, and any Cartier divisor on $\mathcal{X}$ supported in $C$ is a linear combination of $C_{1}, \ldots, C_{t}$. We say that the collection $\left.\mathcal{O}_{\mathcal{X}}\left(C_{1}\right)\right|_{C}, \ldots,\left.\mathcal{O}_{\mathcal{X}}\left(C_{t}\right)\right|_{C}$ is (an ordered basis of) an enriched structure on $C$; cf. [7. Notice that, since $C_{1}+\cdots+C_{t}$ is the special fiber, $\mathcal{O}_{\mathcal{X}}\left(C_{1}+\cdots+C_{n}\right) \cong \mathcal{O}_{\mathcal{X}}$. This allows us to compute the self-intersection of $C_{i}$. For each $i=1, \ldots, t$, letting $\widehat{C}_{i}:=\overline{C-C_{i}}$, we get

$$
\left.\mathcal{O}_{\mathcal{X}}\left(C_{i}\right)\right|_{C_{i}} \cong \mathcal{O}_{C_{i}}\left(-\Delta_{i}\right) \text { and }\left.\mathcal{O}_{\mathcal{X}}\left(C_{i}\right)\right|_{\widehat{C}_{i}} \cong \mathcal{O}_{\widehat{C}_{i}}\left(\Delta_{i}\right) .
$$

For each regular smoothing $f: \mathcal{X} \rightarrow S$ of $C$, let $\mathcal{K}_{f}$ denote the relative dualizing sheaf of $f$. Put $\mathcal{K}_{f}^{\beta}:=\mathcal{K}_{f}^{\otimes \beta}$. The restriction, $\mathcal{K}_{f}^{\beta}(\eta)$ or $\mathcal{K}_{f}^{\beta}(s)$, of $\mathcal{K}_{f}^{\beta}$ to a fibre is the $\beta$-canonical sheaf of that fibre. Since $C$ is nodal, $\mathcal{K}_{f}^{\beta}$ is an invertible sheaf. The sheaves

$$
\mathcal{L}_{f}^{n}:=\mathcal{K}_{f}^{\beta} \otimes \mathcal{O}_{\mathcal{X}}\left(n_{1} C_{1}+\cdots+n_{t} C_{t}\right)
$$

for all $t$-tuples of integers $\underline{n}=\left(n_{1}, \ldots, n_{t}\right)$, are all the invertible extensions of $\mathcal{K}_{f}^{\beta}(\eta)$ to $\mathcal{X}$.

For each $t$-uple $\underline{n}:=\left(n_{1}, \ldots, n_{t}\right)$ of integers, $I \subseteq\{1, \ldots, t\}$ will be called $\underline{n}$ balanced if $n_{i}$ is constant for $i \in I$; if so, let $n_{I}:=n_{i}$ for any $i \in I$. The integer $n_{I}$ is called the $\underline{n}$-weight of $I$. Any nonempty subset $I \subseteq\{1, \ldots, t\}$ is uniquely decomposed into maximal connected $\underline{n}$-balanced subsets. We call these subsets the $\underline{n}$-components of $I$.

Put $L_{f}^{\frac{n}{f}}:=\left.\mathcal{L} \frac{n}{f}\right|_{C}$. For each nonempty subset $I \subseteq\{1, \ldots, t\}$, let $L_{\frac{f}{f, I}}:=\left.L_{\frac{n}{f}}\right|_{C_{I}}$. If $I$ is $\underline{n}$-balanced, it follows from (11) and (4) that

$$
L_{f, I}^{n} \cong K_{I}^{\beta}\left(\sum_{i \in I} \sum_{j \notin I}\left(\beta+n_{j}-n_{i}\right) \Delta_{i, j}\right) .
$$


For each pair $I, J \subset\{1, \ldots, t\}$ of disjoint nonempty subsets, let

$$
\Delta_{I, J}:=\sum_{i \in I} \sum_{j \in J} \Delta_{i, j}
$$

and put $\delta_{I, J}:=\operatorname{deg} \Delta_{I, J}$. For each nonempty, proper subset $I$ of $\{1, \ldots, t\}$, let $I^{c}:=\{1, \ldots, t\}-I$, and put $\Delta_{I}:=\Delta_{I, I^{c}}$ and $\delta_{I}:=\operatorname{deg} \Delta_{I}$.

\section{Spaces of sections of general sheaves}

Lemma 1. Let $X$ be a projective, reduced curve defined over an algebraically closed field $k$ of characteristic zero. Let $D \subset X$ be a collection of nodes and $\pi: \widetilde{X} \rightarrow X$ the partial normalization of $X$ along $D$. Let $\widetilde{L}$ be an invertible sheaf on $\widetilde{X}$ and $L$ the general invertible sheaf on $X$ such that $\pi^{*} L \cong \widetilde{L}$. If, for each $p \in D$, there is an $s \in H^{0}(X, L)$ such that $s(p) \neq 0$, then

$$
h^{0}(X, L)=h^{0}(\widetilde{X}, \widetilde{L})-\# D .
$$

Proof. Let

$$
T:=\operatorname{ker}\left(H^{1}\left(X, \mathcal{O}_{X}\right) \rightarrow H^{1}\left(\widetilde{X}, \mathcal{O}_{\widetilde{X}}\right)\right)
$$

We claim first that the natural bilinear map,

$$
j: H^{0}(X, L) \times T \rightarrow H^{1}(X, L),
$$

is zero; cf. 8, pp. 167-169. Indeed, let $\Phi: \operatorname{Pic}(X) \rightarrow \operatorname{Pic}(\widetilde{X})$ denote the pullback map from the Picard scheme of $X$ to that of $\widetilde{X}$, and put $S:=\Phi^{-1}([\widetilde{L}])$. Since $\Phi$ is a map of group schemes, $S$ is nonsingular. Also, $[L] \in S$. Identify $T$ with the tangent space to $S$ at $[L]$, that is, the set of pointed maps $M$ from $(B, 0)$ to $(S,[L])$, where $B:=\operatorname{Spec}\left(k[t] /\left(t^{2}\right)\right)$ and 0 is the unique point of $B$.

Let $\mathcal{U}$ be the universal invertible sheaf on $X \times S$. Given $[v] \in M$, we have the natural exact sequence

$$
0 \rightarrow L \rightarrow v_{X}^{*} \mathcal{U} \rightarrow L \rightarrow 0
$$

where $v_{X}:=(1, v): X \times B \rightarrow X \times S$. The restriction of $j$ to $H^{0}(X, L) \times\{[v]\}$ is identified with the connecting map $\partial_{v}: H^{0}(X, L) \rightarrow H^{1}(X, L)$ in the associated long exact sequence in cohomology. Now, there is an open dense subset $S_{0} \subseteq S$ such that $h^{0}(X, N)$ is constant for $[N] \in S_{0}$. Since $S_{0}$ is nonsingular, and in particular reduced, the cohomology of $\left.\mathcal{U}\right|_{X \times S_{0}}$ commutes with base change. Thus, for each $[N] \in S_{0}$ and each affine open neighborhood $A$ of $[N]$ in $S_{0}$, the restriction

$$
H^{0}\left(X \times A,\left.\mathcal{U}\right|_{X \times A}\right) \rightarrow H^{0}(X, N)
$$

is surjective. Now, since $L$ is general, $[L] \in S_{0}$. The map $v: B \rightarrow S$ factors through any affine open neighborhood of $[L]$ in $S_{0}$. Thus the restriction map

$$
H^{0}\left(X \times B, v_{X}^{*} \mathcal{U}\right) \rightarrow H^{0}(X, L)
$$

is surjective, and hence the connecting homomorphism $\partial_{v}$ is zero. Since $v$ was arbitrary, $j$ is zero.

Now, let $K$ be the dualizing sheaf of $X$, and $\widetilde{K}$ that of $\widetilde{X}$. Using Serre duality, the dual vector space $T^{*}$ is the cokernel of the natural map $H^{0}\left(X, \pi_{*} \widetilde{K}\right) \rightarrow H^{0}(X, K)$. Also, moving $T$ to the target and $H^{1}(X, L)$ to the source, by taking duals, $j$ is equivalent to the composition of the natural bilinear map,

$$
\gamma: H^{0}(X, L) \times \operatorname{Hom}(L, K) \rightarrow H^{0}(X, K),
$$


with the quotient map to $T^{*}$. Thus, the vanishing of $j$ implies that $\gamma$ factors through $H^{0}\left(X, \pi_{*} \widetilde{K}\right)$.

Let $\phi \in \operatorname{Hom}(L, K)$. Then $\phi(s)$ is a section of $\pi_{*} \widetilde{K}$ for each $s \in H^{0}(X, L)$. Equivalently, since $D$ is a collection of nodes of $X$, and $\widetilde{X}$ is the partial normalization of $X$ along $D$, we have $\phi(s)(p)=0$ for each $p \in D$. Now, by hypothesis, for each $p \in D$ there is an $s \in H^{0}(X, L)$ such that $s(p) \neq 0$. Then $\phi(p)=0$ for each $p \in D$ or, equivalently, $\phi$ factors through $\pi_{*} \widetilde{K}$. Since $\phi$ was arbitrary,

$$
\operatorname{Hom}(L, K)=\operatorname{Hom}\left(L, \pi_{*} \widetilde{K}\right)=\operatorname{Hom}\left(\pi^{*} L, \widetilde{K}\right) .
$$

Thus, using Serre duality and the finiteness of $\pi$,

$$
H^{1}(X, L)=H^{1}\left(\widetilde{X}, \pi^{*} L\right)=H^{1}\left(X, \pi_{*} \pi^{*} L\right) .
$$

Taking cohomology of the natural exact sequence

$$
0 \rightarrow L \rightarrow \pi_{*} \pi^{*} L \rightarrow \bigoplus_{p \in D} k(p) \rightarrow 0
$$

we get

$$
h^{0}(X, L)=h^{0}\left(X, \pi_{*} \pi^{*} L\right)-\# D=h^{0}(\widetilde{X}, \widetilde{L})-\# D,
$$

as we wished to show.

Example. The generality of $L$ is a necessary condition for Lemma 1. Indeed, if $X$ is an irreducible curve with a node $p$, and $\widetilde{X}$ is the partial normalization of $X$ at $p$, then $h^{0}\left(X, \mathcal{O}_{X}\right)=h^{0}\left(\widetilde{X}, \mathcal{O}_{\widetilde{X}}\right)=1$.

Lemma 2. Let $X$ be a projective, reduced curve defined over an algebraically closed field $k$ of characteristic zero. Let $D_{1}$ and $D_{2}$ be two collections of nodes on $X$. Let $X_{1}$ and $X_{2}$ denote the partial normalizations of $X$ along $D_{1}$ and $D_{2}$, and $X_{1,2}$ the partial normalization along $D_{1} \cup D_{2}$. Let $L_{1}$ and $L_{2}$ be two invertible sheaves on $X_{1}$ and $X_{2}$ whose pullbacks to $X_{1,2}$ agree. Then there is an invertible sheaf $L$ on $X$ pulling back to $L_{1}$ on $X_{1}$ and $L_{2}$ on $X_{2}$.

Proof. Let $D:=D_{1} \cap D_{2}$, and $Y$ be the partial normalization of $X$ along $D$. Let $D_{1}^{\prime}:=D_{1}-D$ and $D_{2}^{\prime}:=D_{2}-D$. Then $X_{1}$ and $X_{2}$ are the partial normalizations of $Y$ along $D_{1}^{\prime}$ and $D_{2}^{\prime}$, and $X_{1,2}$ is the partial normalization along $D_{1}^{\prime} \cup D_{2}^{\prime}$. Taking the long exact sequences in cohomology associated to the natural commutative diagram of short exact sequences,

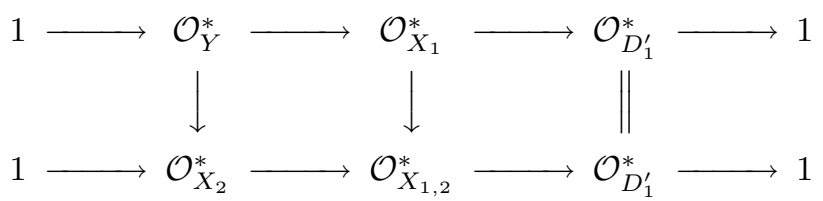

we get

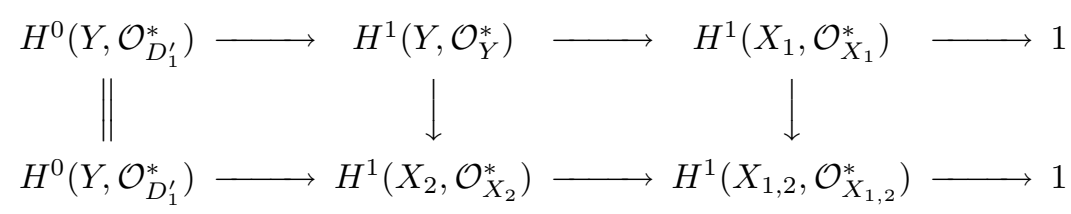

Doing a diagram chase above, we get an invertible sheaf $L^{\prime}$ on $Y$ pulling back to $L_{1}$ on $X_{1}$ and $L_{2}$ on $X_{2}$. Now, since the pullback map $H^{1}\left(X, \mathcal{O}_{X}^{*}\right) \rightarrow H^{1}\left(Y, \mathcal{O}_{Y}^{*}\right)$ is surjective, there is an invertible sheaf $L$ on $X$ pulling back to $L^{\prime}$ on $Y$. 


\section{General conditions}

Recall the notation in Section 2. Let $\underline{n}=\left(n_{1}, \ldots, n_{t}\right)$ be a $t$-tuple of integers. Wherever we assume that the components of $C$ intersect at points in $\underline{n}$-general position, it is to be understood that, for each effective divisor $D=\sum_{i<j} \bar{D}_{i, j}$, with $0 \leq D_{i, j} \leq \Delta_{i, j}$ for all $i$ and $j$, and each $\underline{n}$-balanced subset $I \subseteq\{1, \ldots, t\}$,

$$
h^{0}\left(C_{I}, K_{I}^{\beta}\left(\sum_{i \in I} \sum_{j \notin I}\left(\left(\beta+n_{j}-n_{i}\right) \Delta_{i, j}-D_{i, j}\right)\right)\right)
$$

is the minimum possible, given the components $C_{1}, \ldots, C_{t}$ and the numerical invariants $\delta_{i, j}$. By upper semicontinuity, the condition is indeed general.

Let $I \subseteq\{1, \ldots, t\}$ be a connected subset. Since $C_{I}$ is connected, $h^{0}\left(C_{I}, K_{I}\right)=g_{I}$ and $h^{1}\left(C_{I}, K_{I}\right)=1$. Also, from the Riemann-Roch theorem,

$$
h^{0}\left(C_{I}, K_{I}(E)\right)=g_{I}+\operatorname{deg} E-1
$$

for every effective nonzero divisor $E$ on $C_{I}$ supported in the nonsingular locus.

Furthermore, if $g_{i} \geq 2$ for each $i \in I$, then the sheaf $K_{I}$ is ample. Thus, by Serre duality, $h^{1}\left(C_{I}, K_{I}^{\beta}\right)=0$ for $\beta>1$. From the Riemann-Roch theorem,

$$
h^{0}\left(C_{I}, K_{I}^{\beta}(E)\right)=(2 \beta-1)\left(g_{I}-1\right)+\operatorname{deg} E
$$

for every effective divisor $E$ on $C_{I}$ supported in the nonsingular locus.

For ordering purposes, for each $t$-tuple of integers $\underline{n}=\left(n_{1}, \ldots, n_{t}\right)$ and each nonempty $I \subseteq\{1, \ldots, t\}$, let $m \frac{n}{I}:=\max \left\{n_{i} \mid i \in I\right\}$. Set $m \frac{n}{\emptyset}:=-\infty$. For each nonempty $I \subseteq\{1, \ldots, t\}$, set $\gamma \frac{n}{I}:=0$ if $m_{I^{c}}^{n} \geq m_{I}^{\frac{n}{I}}$ and $\gamma_{I}^{\frac{n}{I}}:=1$ otherwise.

For each subset $I \subseteq\{1, \ldots, t\}$, let $\underline{h}^{I}:=\left(h_{1}^{I}, \ldots, h_{t}^{I}\right)$ be the $t$-tuple given by $h_{i}^{I}:=1$ if $i \in I$ and $h_{i}^{I}:=0$ otherwise.

Lemma 3. Recall the setup and notation of Section 2. Let $\underline{n}=\left(n_{1}, \ldots, n_{t}\right)$ be attuple of integers. Assume that the components of $C$ intersect at points in $\underline{n}$-general position. Let $f: \mathcal{X} \rightarrow S$ be a smoothing of $C$ along the general direction. For $\beta=1$, assume $\delta_{i, j} \neq 0$ for all $i$ and $j$; otherwise assume $g_{i} \geq 2$ for all $i$. For each $i$ and $j$, let $D_{i, j} \leq \Delta_{i, j}$ be an effective divisor. Let $I$ be a nonempty subset of $\{1, \ldots, t\}$, and put

$$
L:=L_{f, I}^{n}\left(-\sum_{i \in I} \sum_{j \notin I} D_{i, j}\right) .
$$

If, for each $i \in I$, the restriction map $H^{0}\left(C_{I}, L\right) \rightarrow H^{0}\left(C_{i},\left.L\right|_{C_{i}}\right)$ is nonzero, then

$$
\begin{cases}h^{1}\left(C_{I}, L\right)=0 & \text { for } \beta>1, \\ h^{1}\left(C_{I}, L\right) \leq \gamma^{\frac{n}{I}}+\underline{h}^{I} & \text { for } \beta=1 .\end{cases}
$$

Proof. Notice that the smoothing $f$ is regular because it is taken along the general direction. We will first treat the special case where $I$ is $\underline{n}$-balanced and connected. In this case, we need only that the smoothing be regular.

Indeed, recall Expression (5) from Section 2:

$$
L_{f, I}^{n} \cong K_{I}^{\beta}\left(\sum_{i \in I} \sum_{j \notin I}\left(\beta+n_{j}-n_{i}\right) \Delta_{i, j}\right) .
$$


Let $P$ and $N$ be effective divisors on $C_{I}$ with disjoint supports such that

$$
\sum_{i \in I} \sum_{j \notin I}\left(\beta+n_{j}-n_{i}\right) \Delta_{i, j}-\sum_{i \in I} \sum_{j \notin I} D_{i, j}=P-N .
$$

Then $L \cong K_{I}^{\beta}(P-N)$.

Clearly,

$$
h^{1}\left(C_{I}, K_{I}^{\beta}(P)\right) \leq h^{1}\left(C_{I}, K_{I}^{\beta}\right) .
$$

If $\beta>1$, then $h^{1}\left(C_{I}, K_{I}^{\beta}\right)=0$, and thus also $h^{1}\left(C_{I}, K_{I}^{\beta}(P)\right)=0$. Now, suppose for the moment that $\beta=1$. Then $h^{1}\left(C_{I}, K_{I}^{\beta}\right)=1$, and thus $h^{1}\left(C_{I}, K_{I}^{\beta}(P)\right) \leq 1$ with equality only if $P=0$. However, from the expression (6), and the hypothesis that $\delta_{i, j} \neq 0$ for all $i$ and $j$, we see that $P=0$ only if $n_{j} \leq n_{i}$ for all $i \in I$ and $j \notin I$, and hence only if $\gamma^{\frac{n}{I}}+\underline{h}^{I}=1$. So

$$
\begin{cases}h^{1}\left(C_{I}, K_{I}^{\beta}(P)\right)=0 & \text { for } \beta>1, \\ h^{1}\left(C_{I}, K_{I}^{\beta}(P)\right) \leq \gamma \frac{n}{I}+\underline{h}^{I} & \text { for } \beta=1 .\end{cases}
$$

It is thus enough to show that

$$
h^{1}\left(C_{I}, L\right)=h^{1}\left(C_{I}, K_{I}^{\beta}(P)\right) .
$$

By the Riemann-Roch theorem, (17) is equivalent to

$$
h^{0}\left(C_{I}, L\right)=h^{0}\left(C_{I}, K_{I}^{\beta}(P)\right)-\operatorname{deg} N .
$$

(Notice that, if $I=\{1, \ldots, t\}$, then $P=N=0$, and the statement of the lemma is proved.)

Write $|N|=\left\{p_{1}, \ldots, p_{s}\right\}$; so $N=\sum_{\ell} w_{\ell} p_{\ell}$ with all $w_{\ell}>0$. For each $\ell=1, \ldots, s$, let $i_{\ell} \in I$ such that $p_{\ell} \in C_{i_{\ell}}$, and pick a general point $q_{\ell} \in C_{i_{\ell}}$ contained in the nonsingular locus of $C_{I}$. Let $\widetilde{N}=\sum_{\ell} w_{\ell} q_{\ell}$ and $\widetilde{L}:=K_{I}^{\beta}(P-\widetilde{N})$.

Since the irreducible components of $C$ intersect at points in $\underline{n}$-general position, $h^{0}\left(C_{I}, L\right) \leq h^{0}\left(C_{I}, \widetilde{L}\right)$. By semicontinuity, the reverse inequality holds. Thus

$$
h^{0}\left(C_{I}, \widetilde{L}\right)=h^{0}\left(C_{I}, L\right) .
$$

For each $i \in I$, since $I-\{i\}$ is $\underline{n}$-balanced, the same reasoning that led to (8) yields

$$
h^{0}\left(C_{I-\{i\}},\left.\widetilde{L}\right|_{C_{I-\{i\}}}\left(-\Delta_{I-\{i\}, i}\right)\right)=h^{0}\left(C_{I-\{i\}},\left.L\right|_{C_{I-\{i\}}}\left(-\Delta_{I-\{i\}, i}\right)\right) .
$$

By hypothesis, the restriction maps $H^{0}\left(C_{I}, L\right) \rightarrow H^{0}\left(C_{i},\left.L\right|_{C_{i}}\right)$ are nonzero for each $i \in I$. Equivalently,

$$
h^{0}\left(C_{I-\{i\}},\left.L\right|_{C_{I-\{i\}}}\left(-\Delta_{I-\{i\}, i}\right)\right)<h^{0}\left(C_{I}, L\right) .
$$

Thus, also the restriction maps

$$
H^{0}\left(C_{I}, \widetilde{L}\right) \rightarrow H^{0}\left(C_{i},\left.\widetilde{L}\right|_{C_{i}}\right) \text { are nonzero for each } i \in I .
$$

So, there is an open dense subset $U \subseteq C_{I}$ contained in the nonsingular locus of $C_{I}$ such that, for $q_{\ell} \in U \cap C_{i_{\ell}}$ for $\ell=1, \ldots, s$, both (8) and (9) hold for $\widetilde{L}:=K_{I}^{\beta}(P-\widetilde{N})$, where $\widetilde{N}=\sum_{\ell} w_{\ell} q_{\ell}$. It is now enough to show that $h^{0}\left(C_{I}, \widetilde{L}\right)=$ $h^{0}\left(C_{I}, K_{I}^{\beta}(P)\right)-\operatorname{deg} \widetilde{N}$ for one such $\widetilde{N}$.

Let $b \leq s$ be a positive integer. Suppose we have already chosen $q_{\ell} \in U \cap C_{i_{\ell}}$ for all $\ell<b$ such that

$$
h^{0}\left(C_{I}, K_{I}^{\beta}(P-E)\right)=h^{0}\left(C_{I}, K_{I}^{\beta}(P)\right)-\operatorname{deg} E,
$$


where $E:=\sum_{\ell<b} w_{\ell} q_{\ell}$. It is enough to show that there is $q_{b} \in U \cap C_{i_{b}}$ such that

$$
h^{0}\left(C_{I}, K_{I}^{\beta}\left(P-E-w_{b} q_{b}\right)\right)=h^{0}\left(C_{I}, K_{I}^{\beta}(P-E)\right)-w_{b} .
$$

Choose $q_{b} \in U \cap C_{i_{b}}$ such that $q_{b}$ is not a ramification point of the linear system of sections of $\left.K_{I}^{\beta}(P-E)\right|_{C_{i_{b}}}$ spanned by $H^{0}\left(C_{I}, K_{I}^{\beta}(P-E)\right)$. Now, if $r_{\ell} \in U \cap C_{i_{\ell}}$ for $\ell=b+1, \ldots, s$, and $F:=\sum_{\ell>b} w_{\ell} r_{\ell}$, then (9) holds for $\widetilde{L}:=K_{I}^{\beta}\left(P-E-w_{b} q_{b}-F\right)$ and $i=i_{b}$. Consequently, the restriction map

$$
H^{0}\left(C_{I}, K_{I}^{\beta}\left(P-E-w_{b} q_{b}\right)\right) \rightarrow H^{0}\left(C_{i_{b}},\left.K_{I}^{\beta}\left(P-E-w_{b} q_{b}\right)\right|_{C_{i_{b}}}\right)
$$

is also nonzero. Given our choice of $q_{b}$, it follows that (10) holds.

For the general case, let $I_{1}, \ldots, I_{p}$ be the $\underline{n}$-components of $I$. For each distinct $i, j \in\{1, \ldots, p\}$, let $E_{i, j} \leq \Delta_{I_{i}, I_{j}}$ be the effective divisor supported on the set of nodes where all sections in $H^{0}\left(C_{I}, L\right)$ vanish. Let $e_{i, j}:=\operatorname{deg} E_{i, j}$ for each $i$ and $j$. Let $E_{i}:=\sum_{j \neq i} E_{i, j}$ and $e_{i}:=\operatorname{deg} E_{i}$ for each $i=1, \ldots, p$. Let $E:=\sum_{i<j} E_{i, j}$ and $e:=\operatorname{deg} E$. Note that $2 e=e_{1}+\cdots+e_{p}$.

Let $\psi: X \rightarrow C_{I}$ be the partial normalization of $C_{I}$ along $|E|$. Let $M:=$ $\psi^{*} L(-F)$ where $F$ is the reduced divisor on $X$ with support $\psi^{-1}(|E|)$. Let $\tilde{X}$ be the disjoint union of the $C_{I_{i}}$ for $i=1, \ldots, p$, and $\pi: \widetilde{X} \rightarrow X$ the natural map.

For each $\underline{n}$-balanced $J \subseteq\{1, \ldots, t\}$, set

$$
L_{J}:=K_{J}^{\beta}\left(\sum_{i \in J} \sum_{j \notin J}\left(\beta+n_{j}-n_{i}\right) \Delta_{i, j}\right) .
$$

Since the smoothing occurs along a general direction, it follows from [6], Cor. 6.9, p. 297 that $L \frac{n}{f}$ is the general invertible sheaf whose restriction to $C_{J}$ is isomorphic to $L_{J}$ for each $\underline{n}$-balanced $J \subseteq\{1, \ldots, t\}$. We will apply Lemma 2 to $C$ and the two collection of nodes, $N_{1}:=\left|\Delta_{I}\right|$ and $N_{2}:=\bigcup\left|\Delta_{J}\right|$, for $J$ running through the $\underline{n}$-components of $\{1, \ldots, t\}$. Their union is

$$
N:=\left(\bigcup_{i=1}^{p}\left|\Delta_{I_{i}}\right|\right) \cup\left(\bigcup_{j=1}^{p^{\prime}}\left|\Delta_{I_{j}^{c}}\right|\right)
$$

where $I_{1}^{c}, \ldots, I_{p^{\prime}}^{c}$ are the $\underline{n}$-components of $I^{c}$. Let $C_{1}$ be the partial normalization of $C$ along $N_{1}$, which is the disjoint union $C_{I} \cup C_{I^{c}}$, and $C_{2}$ the partial normalization of $C$ along $N_{2}$, which is the disjoint union $\bigcup C_{J}$. The partial normalization of $C$ along $N$ is the disjoint union $\widetilde{X} \cup \widetilde{X}^{c}$, where $\widetilde{X}^{c}$ is the disjoint union of the $C_{I_{j}^{c}}$. Let $L_{2}$ be the (unique) invertible sheaf on $C_{2}$ whose restriction to each $C_{J}$ is $L_{J}$. Let $L_{1}$ be the general invertible sheaf on $C_{1}$ whose restrictions to each $C_{I_{i}}$ and each $C_{I_{j}^{c}}$ is $L_{I_{i}}$ and $L_{I_{j}^{c}}$. Notice that each $I_{i}$ is contained in a unique $\underline{n}$-component $J$ of $\{1, \ldots, t\}$, and $\left.L_{J}\right|_{C_{I_{i}}} \cong L_{I_{i}}$. The same holds for each $I_{j}^{c}$. By Lemma 2, there is an invertible sheaf on $C$ pulling back to $L_{1}$ and $L_{2}$. Since $L_{1}$ is general, so is this sheaf, and hence this sheaf is isomorphic to $L \frac{n}{f}$. Since $\left.L_{1}\right|_{C_{I}} \cong L_{f, I}^{\frac{n}{f},}$, and since $L_{1}$ is general, it follows that $L$ is the general invertible sheaf on $C_{I}$ such that

$$
\left.L\right|_{I_{I_{i}}} \cong K_{I_{i}}^{\beta}\left(\sum_{j \in I_{i}} \sum_{\ell \notin I_{i}}\left(\beta+n_{\ell}-n_{j}\right) \Delta_{j, \ell}-\sum_{j \in I_{i}} \sum_{\ell \notin I} D_{j, \ell}\right)
$$


for each $i=1, \ldots, p$. Since the pullback $\psi^{*}: \operatorname{Pic}\left(C_{I}\right) \rightarrow \operatorname{Pic}(X)$ is surjective, it follows that $M$ is the general invertible sheaf on $X$ such that

$$
\pi^{*} M \cong \bigoplus_{i=1}^{p} K_{I_{i}}^{\beta}\left(\sum_{j \in I_{i}} \sum_{\ell \notin I_{i}}\left(\beta+n_{\ell}-n_{j}\right) \Delta_{j, \ell}-\sum_{j \in I_{i}} \sum_{\ell \notin I} D_{j, \ell}-E_{i}\right) .
$$

From the projection formula,

$$
H^{0}\left(C_{I}, L\right)=H^{0}(X, M)
$$

Thus, for each $q \in X$ with $\psi(q) \in \bigcup_{j \neq i}\left|\Delta_{I_{i}, I_{j}}\right|-|E|$, there is a section of $H^{0}(X, M)$ not vanishing at $q$. Applying Lemma 1 to the map $\pi: \widetilde{X} \rightarrow X$ and the sheaf $M$, it follows that

$$
h^{0}\left(C_{I}, L\right)=\sum_{i=1}^{p} h^{0}\left(C_{I_{i}},\left.L\right|_{C_{I_{i}}}\left(-E_{i}\right)\right)-\sum_{i<j} \delta_{I_{i}, I_{j}}+e .
$$

Suppose first that $\beta>1$. For each $i=1, \ldots, p$, replacing $I$ by $I_{i}$ and $L$ by $\left.L\right|_{C_{I_{i}}}\left(-E_{i}\right)$ in the statement of the lemma, we see that its hypotheses hold. Indeed, $\left.L\right|_{C_{I_{i}}}\left(-E_{i}\right)=L \frac{n}{f, I_{i}}(-P)$ for a certain effective reduced divisor $P$ whose support is contained in the set of nodes of $C$ that are nonsingular points of $C_{I_{i}}$. Also, since all sections of $H^{0}\left(C_{I}, L\right)$ vanish on $E_{i}$, for each $j \in I_{i}$, any section of $H^{0}\left(C_{I}, L\right)$ that does not vanish on $C_{j}$ restricts to a section of $H^{0}\left(C_{I_{i}},\left.L\right|_{C_{I_{i}}}\left(-E_{i}\right)\right)$ with the same property. Now, $I_{i}$ is $\underline{n}$-balanced and connected. As the lemma was already proved in this special case, we may apply it, to get

$$
h^{1}\left(C_{I_{i}},\left.L\right|_{C_{I_{i}}}\right)=h^{1}\left(C_{I_{i}},\left.L\right|_{C_{I_{i}}}\left(-E_{i}\right)\right)=0 .
$$

So, by the Riemann-Roch theorem,

$$
h^{0}\left(C_{I_{i}},\left.L\right|_{C_{I_{i}}}\left(-E_{i}\right)\right)=h^{0}\left(C_{I_{i}},\left.L\right|_{C_{I_{i}}}\right)-e_{i}
$$

for each $i=1, \ldots, p$. Using (11), we get

$$
h^{0}\left(C_{I}, L\right)=\sum_{i=1}^{p} h^{0}\left(C_{I_{i}},\left.L\right|_{C_{I_{i}}}\right)-\sum_{i<j} \delta_{I_{i}, I_{j}}-e .
$$

However, it follows from the long exact sequence in cohomology associated to the short exact sequence

$$
\left.0 \rightarrow L \rightarrow \bigoplus_{i=1}^{p} L\right|_{C_{I_{i}}} \rightarrow \bigoplus_{i<j} \bigoplus_{q \in\left|\Delta_{I_{i}, I_{j}}\right|} k(q) \rightarrow 0
$$

that

$$
h^{0}\left(C_{I}, L\right) \geq \sum_{i=1}^{p} h^{0}\left(C_{I_{i}},\left.L\right|_{C_{I_{i}}}\right)-\sum_{i<j} \delta_{I_{i}, I_{j}},
$$

with equality if and only if

$$
h^{1}\left(C_{I}, L\right)=\sum_{i=1}^{p} h^{1}\left(C_{I_{i}},\left.L\right|_{C_{I_{i}}}\right) .
$$

So equality holds, and hence $h^{1}\left(C_{I}, L\right)=0$, as we wished to show.

Now, suppose $\beta=1$. Again, apply the statement of the lemma to $\left.L\right|_{C_{I_{i}}}\left(-E_{i}\right)$, this time to get

$$
h^{1}\left(C_{I_{i}},\left.L\right|_{C_{I_{i}}}\left(-E_{i}\right)\right) \leq \gamma \bar{n}_{i_{i}} \underline{h}^{I_{i}}
$$


so, by the Riemann-Roch theorem,

$$
h^{0}\left(C_{I_{i}},\left.L\right|_{C_{I_{i}}}\left(-E_{i}\right)\right) \leq h^{0}\left(C_{I_{i}},\left.L\right|_{C_{I_{i}}}\right)-e_{i}+\gamma_{\bar{I}_{i}}^{\underline{n}} \underline{\underline{h}}^{I_{i}}
$$

for each $i=1, \ldots, p$. From (11) we get

$$
h^{0}\left(C_{I}, L\right) \leq \sum_{i=1}^{p} h^{0}\left(C_{I_{i}},\left.L\right|_{C_{I_{i}}}\right)-\sum_{i<j} \delta_{I_{i}, I_{j}}-e+\sum_{i=1}^{p} \gamma{\frac{n}{I_{i}}}^{+\underline{h}^{I_{i}}} .
$$

Observe now that

$$
\sum_{i=1}^{p} \gamma \frac{n}{I_{i}} \underline{\underline{h}}^{I_{i}}=\gamma \gamma^{\frac{n}{I}} \underline{\underline{h}}^{I}
$$

Indeed, since, by hypothesis, each two components of $C_{I}$ intersect, the $\underline{n}$-weights of the $I_{i}$ are different. So, there is $j \in\{1, \ldots, p\}$ such that $m \frac{n}{I_{j}}>m \frac{n}{I_{i}}$ for each $i \neq j$. Hence $\gamma \bar{n}^{n} \underline{\underline{h}}^{I_{i}}=0$ for each $i \neq j$. In addition, $\gamma^{\frac{n}{I_{j}}+\underline{h}^{I_{j}}}=0$ if and only if $m \frac{n}{I^{c}} \geq m \frac{n}{I_{j}}+1$. Now,

$$
m \frac{n}{I}=\max \left(m \frac{n}{I_{1}}, \ldots, m \frac{n}{I_{p}}\right)=m \frac{n}{I_{j}},
$$

and $\gamma^{\frac{n}{I}}+\underline{h}^{I}=0$ if and only if $m \frac{n}{I^{c}} \geq m \frac{n}{I}+1$. So

$$
\gamma \frac{n}{I}+\underline{h}^{I}=\gamma \frac{n}{I_{j}} \underline{h}^{I_{j}}=\sum_{i=1}^{p} \gamma \frac{n}{I_{i}} \underline{h}^{I_{i}}
$$

as we wished to show.

Combining (13) with (12), we get

$$
h^{0}\left(C_{I}, L\right) \leq \sum_{i=1}^{p} h^{0}\left(C_{I_{i}},\left.L\right|_{C_{I_{i}}}\right)-\sum_{i<j} \delta_{I_{i}, I_{j}}-e+\gamma_{\underline{I}}^{\underline{n} \underline{h}^{I}} .
$$

Since $\gamma^{\frac{n}{I}}+\underline{h}^{I} \leq 1$, if $e>0$ we get

$$
h^{0}\left(C_{I}, L\right) \leq \sum_{i=1}^{p} h^{0}\left(C_{I_{i}},\left.L\right|_{C_{I_{i}}}\right)-\sum_{i<j} \delta_{I_{i}, I_{j}}
$$

The same inequality can be obtained directly from (11) if $e=0$. So, as in the case $\beta>1$, we get

$$
h^{1}\left(C_{I}, L\right)=\sum_{i=1}^{p} h^{1}\left(C_{I_{i}},\left.L\right|_{C_{I_{i}}}\right)
$$

But

$$
\sum_{i=1}^{p} h^{1}\left(C_{I_{i}},\left.L\right|_{C_{I_{i}}}\right) \leq \sum_{i=1}^{p} h^{1}\left(C_{I_{i}},\left.L\right|_{C_{I_{i}}}\left(-E_{i}\right)\right) \leq \sum_{i=1}^{p} \gamma_{I_{i}}^{\underline{n}+\underline{h}^{I_{i}}}=\gamma_{\bar{I}}^{\underline{n}+\underline{h}^{I}},
$$

finishing the proof. 


\section{A NUMERICAL LEMMA}

Let $\Gamma$ be a one-dimensional, connected, unoriented graph with $t$ vertices. Order the vertices. For all distinct $i, j \in\{1, \ldots, t\}$ let $\delta_{i, j}$ be the number of edges between the $i$-th and $j$-th vertices. Let $\delta:=\sum_{i<j} \delta_{i, j}$. For each pair $I, J \subset\{1, \ldots, t\}$ of disjoint nonempty subsets, let

$$
\delta_{I, J}:=\sum_{i \in I} \sum_{j \in J} \delta_{i, j}
$$

For each subset $I$ of $\{1, \ldots, t\}$, let $I^{c}:=\{1, \ldots, t\}-I$.

For each pair of $t$-tuples of integers, $\underline{e}=\left(e_{1}, \ldots, e_{t}\right)$ and $\underline{n}=\left(n_{1}, \ldots, n_{t}\right)$, let

$$
\epsilon_{i}^{\underline{e}, \underline{n}}:=e_{i}+\sum_{j \neq i}\left(n_{j}-n_{i}\right) \delta_{i, j} \text { for each } i=1, \ldots, t .
$$

In addition, for each nonempty $I \subseteq\{1, \ldots, t\}$, let

$$
\epsilon_{I}^{\frac{e}{\underline{n}} \underline{n}}:=\sum_{i \in I} \epsilon_{i}^{\underline{e}, \underline{n}}-\sum_{\substack{i, j \in I \\ i<j}} \delta_{i, j} .
$$

Recall the definitions of $m \frac{n}{I}, \gamma \frac{n}{I}$ and $\underline{h}^{I}$ from Section 4. For each positive integer $\beta$ and each $\ell \in\{1, \ldots, t\}$, let

$$
\gamma_{I}^{\beta, \ell, \underline{n}}:= \begin{cases}\gamma^{\frac{n}{I}} & \text { if } \beta=1, \\ 1 & \text { if } \beta>1 \text { and } \ell \in I, \\ 0 & \text { if } \beta>1 \text { and } \ell \notin I .\end{cases}
$$

Lemma 4. Let $\beta$ be a positive integer. For $\beta=1$, assume $\delta_{i, j} \neq 0$ for all $i$ and $j$. Fix an integer $\ell \in\{1, \ldots, t\}$. Let $\underline{e}=\left(e_{1}, \ldots, e_{t}\right)$ be a t-tuple of integers such that $e_{1}+\cdots+e_{t}=\delta$. Then there is a unique t-tuple of integers $\underline{n}=\left(n_{1}, \ldots, n_{t}\right)$ such that $n_{\ell}=0$, and such that for each proper, nonempty $I \subset\{1, \ldots, t\}$,

$$
\gamma_{I^{c}}^{\beta, \ell, \underline{n}+\underline{h}^{I^{c}}} \leq \epsilon_{I}^{\underline{e}, \underline{n}} \leq \delta_{I, I^{c}}-\gamma_{I}^{\beta, \ell, \underline{n}+\underline{h}^{I}} .
$$

Proof. Without loss of generality, we may assume $\ell=1$. To simplify the notation, put $\epsilon_{I}^{\underline{n}}:=\epsilon_{I}^{e, \underline{n}}$ and $\gamma_{I}^{\beta, \underline{n}}:=\gamma_{I}^{\beta, 1, \underline{n}}$ for each $t$-tuple of integers $\underline{n}$ and each nonempty $I \subseteq\{1, \ldots, t\}$.

First notice that, since $e_{1}+\cdots+e_{t}=\delta$,

$$
\epsilon_{\{1, \ldots, t\}}=0 \text { for each } \underline{n} \text {. }
$$

Also, if a subset $I$ of $\{1, \ldots, n\}$ is the disjoint union of nonempty $I_{1}, \ldots, I_{p}$, then

$$
\epsilon \frac{n}{I}=\sum_{i=1}^{p} \epsilon_{I_{i}}^{\frac{n}{I_{i}}}-\sum_{1 \leq i<j \leq p} \delta_{I_{i}, I_{j}} \text { for each } \underline{n} .
$$

Thus $\epsilon_{I}^{\frac{n}{I}}+\epsilon_{I^{c}}^{\frac{n}{c}}=\delta_{I, I^{c}}$ for each proper, nonempty $I \subset\{1, \ldots, t\}$. It follows that the inequality $\epsilon_{I}^{n} \leq \delta_{I, I^{c}}-\gamma_{I}^{\beta, \underline{n}+\underline{h}^{I}}$ is equivalent to $\epsilon_{I^{c}}^{\underline{n}} \geq \gamma_{I}^{\beta, \underline{n}+\underline{h}^{I}}$. Thus, we need only prove the lemma for every nonempty $I \subseteq\{2, \ldots, t\}$.

We divide the proof in five parts:

Part 1: We claim there is a $t$-tuple $\underline{n}=\left(0, n_{2}, \ldots, n_{t}\right)$ such that $\epsilon_{i}^{\underline{n}}>>0$ for all $i=2, \ldots, t$. To prove our claim, define inductively a partition $\{2, \ldots, t\}=I_{1} \cup$ $\cdots \cup I_{q}$ as follows. Let $I_{1}:=\left\{i \in\{2, \ldots, t\} \mid \delta_{1, i}>0\right\}$; assuming that $I_{r}$ is defined, let $I_{r+1} \subseteq\{2, \ldots, t\}$ be the subset consisting of those $i \in\{2, \ldots, t\}-\left(I_{1} \cup \cdots \cup I_{r}\right)$ such that $\delta_{i, j}>0$ for some $j \in I_{r}$. Since the graph $\Gamma$ is connected, if $I_{1} \cup \cdots \cup I_{r} \neq$ 
$\{2, \ldots, t\}$, then $I_{r+1}$ is nonempty. So, after finitely many steps, say $q$ steps, we obtain a partition of $\{2, \ldots, t\}$.

For each integer $s$, and each $r=1, \ldots, q$, let $\underline{n}^{r, s}=\left(0, n_{2}^{r, s}, \ldots, n_{t}^{r, s}\right)$ be the $t$-tuple of integers defined by letting $n_{i}^{r, s}:=s$ if $i \in I_{r} \cup \cdots \cup I_{q}$, and $n_{i}^{r, s}:=0$ otherwise. For each $q$-tuple $\underline{s}:=\left(s_{1}, \ldots, s_{q}\right)$ of integers, let

$$
\underline{n}^{\underline{s}}:=\sum_{r=1}^{q} \underline{n}^{r, s_{r}} .
$$

Let $I_{0}:=\{1\}, I_{q+1}:=\emptyset$ and $s_{q+1}:=0$. Then, for each $r=1, \ldots, q$, and each $i \in I_{r}$, since by construction $\delta_{i, j} \neq 0$ only if $j \in I_{r-1} \cup I_{r} \cup I_{r+1}$,

$$
\epsilon_{i}^{\underline{n}^{\underline{s}}}=e_{i}-s_{r}\left(\sum_{j \in I_{r-1}} \delta_{i, j}\right)+s_{r+1}\left(\sum_{j \in I_{r+1}} \delta_{i, j}\right) .
$$

Also by construction, $\sum_{j \in I_{r-1}} \delta_{i, j}>0$ for every $i \in I_{r}$, for each $r=1, \ldots, q$. Consequently, we can make $\epsilon_{i}^{\underline{\underline{n}}}>>0$ for every $i \in I_{q}$ by letting $s_{q}<<0$. Assuming that $s_{r+1}, \ldots, s_{q}$ were chosen such that $\epsilon_{i}^{\underline{n}^{\underline{s}}}>>0$ for every $i \in I_{r+1} \cup \cdots \cup I_{q}$, let $s_{r}<<0$ to make $\epsilon_{i}^{\underline{\underline{n}}} \underline{\underline{s}}>>$ for every $i \in I_{r}$. By induction, there are integers $s_{i}<<0$ for $i=1, \ldots, q$ such that $\epsilon_{i}^{\underline{\underline{n}}} \underline{\underline{s}}>>$ for each $i=2, \ldots, t$. The proof of our first claim is complete.

Part 2: For each integer $s \geq 0$, let $\underline{n}^{s}=\left(0, n_{2}^{s}, \ldots, n_{t}^{s}\right)$ be a $t$-tuple of integers. Assume that $n_{i}^{s} \leq n_{i}^{s+1}$ for each $i=2, \ldots, t$ and each $s \geq 0$. In addition, assume that

$$
\lim _{s \rightarrow \infty} n_{i}^{s}=\infty
$$

for a certain $i \in\{2, \ldots, t\}$. We claim that there is $j \in\{2, \ldots, t\}$ such that

$$
\liminf _{s \rightarrow \infty} \epsilon_{j}^{n^{s}}=-\infty
$$

Indeed, up to reordering $\{2, \ldots, t\}$, there is an integer $u$ with $1 \leq u<t$ such that $\lim _{s \rightarrow \infty} n_{j}^{s}=\infty$ if and only if $j>u$. Since

$$
\sum_{j=2}^{t} \epsilon_{j}^{n^{s}}=\sum_{j=2}^{t} e_{j}-\sum_{j=2}^{t} \delta_{1, j} n_{j}^{s},
$$

there is $j \in\{2, \ldots, t\}$ such that (15) holds, unless $\delta_{1, j}=0$ for each $j>u$. Now, suppose $\delta_{1, j}=0$ for each $j>u$. Then $u>1$, because the graph $\Gamma$ is connected. For each $r=2, \ldots, u$, since $\lim _{s \rightarrow \infty} n_{j}^{s}=\infty$ if and only if $j>u$, either $\lim _{s \rightarrow \infty} \epsilon_{r}^{n^{s}}=\infty$ or $\delta_{r, u+1}=\cdots=\delta_{r, t}=0$. However, since $\Gamma$ is connected, we cannot have $\delta_{r, j}=0$ for each $r=1, \ldots, u$ and each $j=u+1, \ldots, t$. Thus $\lim _{s \rightarrow \infty} \epsilon_{r}^{n^{s}}=\infty$ for a certain $r \in\{2, \ldots, u\}$. However, since

$$
\sum_{j=2}^{t} \epsilon_{j}^{\underline{n}^{s}} \leq \sum_{j=2}^{t} e_{j}-\sum_{j=2}^{t} \delta_{1, j} n_{j}^{0}
$$

for each $s \geq 0$, then (15) holds for some $j \neq r$. The proof of our claim is complete.

Part 3: It follows from Part 1 that there is a $t$-tuple $\underline{n}=\left(n_{1}, \ldots, n_{t}\right)$ such that $n_{1}=0$ and

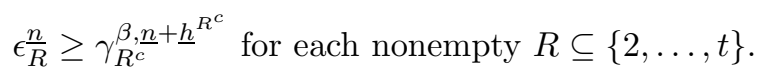


Assume that there is a nonempty subset $I \subseteq\{2, \ldots, t\}$ such that

$$
\epsilon_{I}^{\underline{n}} \geq \delta_{I, I^{c}}-\gamma_{I}^{\beta, \underline{n}+\underline{h}^{I}}+1 \text {. }
$$

Suppose $I$ is minimal with the above property. Let $\underline{n}^{\prime}:=\underline{n}+\underline{h}^{I}$. We claim that (16) holds for $\underline{n}^{\prime}$ in place of $\underline{n}$.

Indeed, let $R \subseteq\{2, \ldots, t\}$ be a nonempty subset. We will prove that

$$
\epsilon \frac{n^{\prime}}{R} \geq \gamma_{R^{c}}^{\beta, \underline{n}^{\prime}}+\underline{h}^{R^{c}}
$$

We divide the proof in three cases:

Case 1: Suppose first that $R \subseteq I$. Suppose for the moment that $R \neq I$, and let $S:=I-R$. Since $I$ is minimal for property (17),

$$
\epsilon \frac{n}{S} \leq \delta_{S, S^{c}}-\gamma_{S}^{\beta, \underline{n}+\underline{h}^{S}} .
$$

Since $I$ is a disjoint union of $R$ and $S$, from (14) we get

$$
\epsilon_{I}^{\frac{n}{I}}=\epsilon_{\frac{n}{R}}+\epsilon_{S}^{\frac{n}{S}}-\delta_{R, S}
$$

and thus, from (17) and (19),

$$
\epsilon_{R}^{\underline{n}} \geq \delta_{R, I^{c}}+\gamma_{S}^{\beta, \underline{n}+\underline{h}^{S}}-\gamma_{I}^{\beta, \underline{n}+\underline{h}^{I}}+1 \text {. }
$$

Note now that

$$
\gamma_{I}^{\beta, \underline{n}+\underline{h}^{I}} \leq \gamma_{R}^{\beta, \underline{n}+\underline{h}^{I}}+\gamma_{S}^{\beta, \underline{n}+\underline{h}^{S}} .
$$

Indeed, if $\beta>1$, since $1 \notin I$, all terms in (21) are zero. So suppose $\beta=1$. Since all the terms in (21) are either 0 or 1 , we may also suppose that $\gamma_{R}^{\frac{n}{R}+\underline{h}^{I}}=0$ and $\gamma \frac{n}{S}+\underline{h}^{S}=0$, and show that $\gamma_{\frac{n}{I}}+\underline{h}^{I}=0$. Equivalently, suppose

$$
m \frac{n}{R}+1 \leq \max \left(m \frac{n}{S}+1, m \frac{n}{I^{c}}\right) \text { and } m \frac{n}{S}+1 \leq \max \left(m \frac{n}{R}, m \frac{n}{I^{c}}\right) .
$$

Coupling the two inequalities, we get $m \frac{n}{R}+1 \leq \max \left(m \frac{n}{R}, m \frac{n}{I^{c}}\right)$. This is only possible if $m \frac{n}{R}+1 \leq m \frac{n}{I^{c}}$. Then $m \frac{n}{S}+1 \leq m \frac{n}{I^{c}}$ as well, and thus

$$
m \frac{n}{I}+1=\max \left(m \frac{n}{R}, m \frac{n}{S}\right)+1 \leq m \frac{n}{I^{c}} .
$$

The latter inequality means $\gamma_{I}^{\underline{n}}+\underline{h}^{I}=0$.

Using (21) in (20), we get

$$
\epsilon \frac{n}{R} \geq \delta_{R, I^{c}}-\gamma_{R}^{\beta, \underline{n}+\underline{h}^{I}}+1 .
$$

Notice that the above inequality still holds for $I$ instead of $R$, as we recover (17).

Thus, we may now drop the hypothesis that $R \neq I$. By (22),

$$
\epsilon_{R}^{n^{\prime}}=\epsilon \frac{n}{R}-\delta_{R, I^{c}} \geq 1-\gamma_{R}^{\beta, \underline{n}+\underline{h}^{I}} .
$$

To get (18), we need only show that

$$
\gamma_{R}^{\beta, \underline{n}+\underline{h}^{I}}+\gamma_{R^{c}}^{\beta, \underline{n}^{\prime}+\underline{h}^{R^{c}}} \leq 1 .
$$

Now, the inequality holds unless both terms on the left are 1 . However, if $\beta>1$, then $\gamma_{R}^{\beta, \underline{n}+\underline{h}^{I}}=0$ because $1 \notin R$. And if $\beta=1$, either $m \frac{\underline{n}^{\prime}}{R^{c}}+1 \leq m \frac{n^{\prime}}{R}$, whence $\gamma \frac{n^{\prime}+\underline{h}^{R^{c}}}{R^{c}}=0$, or $m \frac{n^{\prime}}{R} \leq m \frac{n^{\prime}}{R^{c}}$, and hence $\gamma \frac{n}{R}+\underline{h}^{I}=0$.

Case 2: Suppose now that $R \subseteq I^{c}$. Then, by (16),

$$
\epsilon_{\bar{R}}^{n^{\prime}}=\epsilon_{R}^{n}+\delta_{R, I} \geq \gamma_{R^{c}}^{\beta, \underline{n}+\underline{h}^{R^{c}}}+\delta_{R, I} .
$$


Now,

$$
\gamma_{R^{c}}^{\beta, \underline{n}+\underline{h}^{R^{c}}}+\delta_{R, I} \geq 1
$$

because $\delta_{R, I} \geq 1$ for $\beta=1$, by hypothesis, and because $\gamma_{R^{c}, \underline{n}+\underline{h}^{R^{c}}}^{\beta}=1$ for $\beta>1$, since $1 \in R^{c}$. Thus $\epsilon_{R}^{n^{\prime}} \geq 1 \geq \gamma_{R^{c}}^{\beta, \underline{n}^{\prime}}+\underline{h}^{R^{c}}$.

Case 3: Let $R_{1}:=R \cap I$ and $R_{2}:=R \cap I^{c}$. Suppose $R_{1}$ and $R_{2}$ are nonempty. Then, using (22) with $R_{1}$ in place of $R$, and (16) with $R_{2}$ in place of $R$, we get

$$
\begin{aligned}
\epsilon \frac{n^{\prime}}{R} & =\epsilon \frac{n^{\prime}}{R_{1}}+\epsilon_{R_{2}}^{n^{\prime}}-\delta_{R_{1}, R_{2}} \\
& =\epsilon \frac{n}{R_{1}}-\delta_{R_{1}, I^{c}}+\epsilon \frac{n}{R_{2}}+\delta_{R_{2}, I}-\delta_{R_{1}, R_{2}} \\
& \geq \delta_{R_{1}, I^{c}}-\gamma_{R_{1}}^{\beta, \underline{n}+\underline{h}^{I}}+1-\delta_{R_{1}, I^{c}}+\gamma_{R_{2}^{\beta}}^{\beta, \underline{n}}+\underline{h}^{R_{2}^{c}}+\delta_{R_{2}, I}-\delta_{R_{1}, R_{2}} \\
& \geq 1+\gamma_{R_{2}^{\beta}}^{\beta, \underline{n}+\underline{h}^{R_{2}^{c}}}-\gamma_{R_{1}^{\beta}}^{\beta, \underline{n}}+\underline{h}^{I} .
\end{aligned}
$$

To get (18), we need only show that

$$
\gamma_{R_{1}^{\beta, \underline{n}^{\prime}}}^{\beta}+\gamma_{R^{c}}^{\beta, \underline{n}^{\prime}+\underline{h}^{R^{c}}} \leq \gamma_{R_{2}^{c}}^{\beta, \underline{n}+\underline{h}^{R_{2}^{c}}}+1 .
$$

If $\beta>1$, then $\gamma_{R_{1}}^{\beta, \underline{n}^{\prime}}=0$ because $1 \notin R_{1}$, and hence the inequality holds. So suppose $\beta=1$. We may also suppose $\gamma_{R_{1}}^{\beta, \underline{n^{\prime}}}=1$ and $\gamma_{R^{c}}^{\beta, \underline{n}^{\prime}+\underline{h}^{R^{c}}}=1$, and show that $\gamma_{R_{2}^{c}}^{\beta, \underline{n}+\underline{h}^{R_{2}^{c}}}=1$. Equivalently, suppose

$$
m \frac{n^{\prime}}{R_{1}}>m \frac{n^{\prime}}{R_{1}^{c}} \text { and } m \frac{n^{\prime}}{R^{c}}+1>m \frac{n^{\prime}}{R} .
$$

Using the second inequality in (23) we get

$$
m \frac{n}{R_{2}}=m \frac{n^{\prime}}{R_{2}} \leq m \frac{n^{\prime}}{R} \leq m \frac{n^{\prime}}{R^{c}} .
$$

Also, using the first inequality, $m_{\frac{n}{I}-R_{1}}<m \frac{n}{R_{1}}$, whence $m \frac{n}{I-R_{1}}+1 \leq m \frac{n}{I}$. Since

$$
m \frac{n^{\prime}}{R^{c}}=\max \left(m \frac{n}{I^{c}-R_{2}}, m \frac{n}{I-R_{1}}+1\right),
$$

we get

$$
m \frac{n^{\prime}}{R^{c}} \leq \max \left(m \frac{n}{I^{c}}-R_{2}, m \frac{n}{I}\right)=m \frac{n}{R_{2}^{c}} .
$$

Combining (24) with (25), we get $m \frac{n}{R_{2}} \leq m \frac{n}{R_{2}^{c}}$, which is equivalent to $\gamma_{R_{2}^{c}}^{\beta, \underline{n}+\underline{h}^{R_{2}^{c}}}=1$.

Part 4: In Part 3, we started with a $t$-tuple $\underline{n}=\left(n_{1}, \ldots, n_{t}\right)$ satisfying $n_{1}=0$ and inequalities (16), and produced another $t$-tuple $\underline{n}^{\prime}=\left(n_{1}^{\prime}, \ldots, n_{t}^{\prime}\right)$ satisfying $n_{1}^{\prime}=0$ and the same inequalities, (18), as $\underline{n}$. We may thus apply Part 3 again, with $\underline{n}^{\prime}$ instead of $\underline{n}$, and so on, as long as (17) holds for some nonempty subset $I \subseteq\{2, \ldots, t\}$. However, $n_{i}^{\prime} \geq n_{i}$ for each $i$, with strict inequality for some $i$. Hence, according to Part 2, this process must come to an end. When it does, we obtain a $t$-tuple $\underline{n}$ satisfying all the inequalities stated in the lemma.

Part 5: Finally, suppose that there exist two $t$-tuples, $\underline{n}=\left(0, n_{2}, \ldots, n_{t}\right)$ and $\underline{n}^{\prime}=\left(0, n_{2}^{\prime}, \ldots, n_{t}^{\prime}\right)$, satisfying the inequalities in the statement of the lemma. Let $\underline{x}:=\underline{n}^{\prime}-\underline{n}$. Write $\underline{x}=\left(x_{1}, \ldots, x_{t}\right)$, and put $x:=\max \left(x_{1}, \ldots, x_{t}\right)$. Assume that $\underline{x} \neq 0$. Then, by exchanging $\underline{n}$ and $\underline{n}^{\prime}$ if necessary, we may assume that $x>0$. Let 
$I \subseteq\{2, \ldots, t\}$ be the maximal $\underline{x}$-balanced subset of $\underline{x}$-weight $x$. Then, using the stated left-hand inequalities for $\underline{n}^{\prime}$,

$$
\epsilon_{I}^{\underline{n}}=\epsilon_{I}^{\underline{n}^{\prime}}+\sum_{j \in I^{c}} \delta_{I,\{j\}}\left(x-x_{j}\right) \geq \gamma_{I^{c}}^{\beta, \underline{n}^{\prime}+\underline{h}^{I^{c}}}+\delta_{I, I^{c}}+\sum_{j \in I^{c}} \delta_{I,\{j\}}\left(x-1-x_{j}\right) .
$$

It follows, as we claim, that $\epsilon_{I}^{\underline{n}}>\delta_{I, I^{c}}-\gamma_{I}^{\beta, \underline{n}+\underline{h}^{I}}$, thus reaching a contradiction with the stated right-hand inequalities for $\underline{n}$. Indeed, we need only show that

$$
\gamma_{I^{c}}^{\beta, \underline{n}^{\prime}+\underline{h}^{I^{c}}}+\gamma_{I}^{\beta, \underline{n}+\underline{h}^{I}}+\sum_{j \in I^{c}} \delta_{I,\{j\}}\left(x-1-x_{j}\right)>0 .
$$

However, if the last inequality does not hold, then $\gamma_{I}^{\beta, \underline{n}+\underline{h}^{I}}=\gamma_{I^{c}}^{\beta, \underline{n}^{\prime}+\underline{h}^{I^{c}}}=0$ and $\delta_{I,\{j\}}\left(x-1-x_{j}\right)=0$ for each $j \in I^{c}$. Since $1 \in I^{c}$, the equality $\gamma_{I^{c}}^{\beta, \underline{n}^{\prime}+\underline{h}^{I^{c}}}=0$ implies $\beta=1$. Then, by hypothesis, $\delta_{I,\{j\}}>0$ for each $j \in I^{c}$. So $x_{j}=x-1$ for each $j \in I^{c}$. Since $1 \in I^{c}$ and $x_{1}=0$, we have $\underline{x}=\underline{h}^{I}$. So $\gamma_{I^{c}}^{n}=\gamma_{I^{c}}^{\beta, \underline{n}^{\prime}+\underline{h}^{I^{c}}}$, and hence $\gamma \frac{n}{I^{c}}=0$. This is equivalent to

$$
m \frac{n}{I^{c}} \leq m \frac{n}{I}
$$

On the other hand, $\gamma_{I}^{\beta, \underline{n}+\underline{h}^{I}}=0$ is equivalent to

$$
m \frac{n}{I}+1 \leq m \frac{n}{I^{c}} .
$$

We have a contradiction.

For later use, denote by $\underline{n}^{(\ell)}(\underline{e})$ the unique $t$-tuple of integers $\underline{n}$ of the statement of Lemma 4.

Example. We will show that the condition " $\delta_{i, j} \neq 0$ for all $i$ and $j$ " is necessary for the uniqueness of $\underline{n}$, when $\beta=1$, in Lemma 4

Let $\Gamma$ be a graph with three vertices. Assume that $\delta_{1,2}=\delta_{1,3}=1$ and $\delta_{2,3}=0$. Let $g$ be a positive integer and put $\underline{e}:=(-2 g+2, g, g)$. Let $\ell:=1$.

Let $\underline{n}:=(0, g, g-1)$. We claim that the inequalities in Lemma 4 are satisfied. Indeed, we have $\epsilon_{1}^{\underline{e}, \underline{n}}=\epsilon_{3}^{\underline{e}, \underline{n}}=1$ and $\epsilon_{2}^{\underline{e}, \underline{n}}=0$. If $I=\{1,2\}$, then

$$
\epsilon_{I}^{\underline{e}, \underline{n}}=0, \gamma_{\{3\}}^{(0, g, g)}=0, \gamma_{\{1,2\}}^{(1, g+1, g-1)}=1, \delta_{I, I^{c}}=1 .
$$

If $I=\{2,3\}$, then

$$
\epsilon_{I}^{\underline{e}, \underline{n}}=1, \gamma_{\{1\}}^{(1, g, g-1)}=0, \gamma_{\{2,3\}}^{(0, g+1, g)}=1, \delta_{I, I^{c}}=2 .
$$

If $I=\{1,3\}$, then

$$
\epsilon_{I}^{e, \underline{n}}=1, \gamma_{\{2\}}^{(0, g+1, g-1)}=1, \gamma_{\{1,3\}}^{(1, g, g)}=0, \delta_{I, I^{c}}=1 .
$$

In each case, the inequalities of the lemma hold. As we have observed in the beginning of the proof of the lemma, it follows that the inequalities hold as well for every $I \subset\{1,2,3\}$ with $\# I=1$. So all the inequalities in Lemma 4 are satisfied.

However, because of the symmetry of the numerical invariants of $\Gamma$, also if we set $\underline{n}:=(0, g-1, g)$ the inequalities are satisfied. 


\section{Limit Weierstrass POINTS}

Recall the notation in Section 2. For each $\beta$ define,

$$
\kappa_{\beta}:= \begin{cases}g & \text { if } \beta=1, \\ (2 \beta-1)(g-1) & \text { if } \beta>1 .\end{cases}
$$

For each $\ell=1, \ldots, t$ let $\underline{e}^{(\ell)}=\left(e_{1}^{(\ell)}, \ldots, e_{t}^{(\ell)}\right)$ be the $t$-tuple of integers defined by letting

$$
e_{j}^{(\ell)}:= \begin{cases}(2 \beta-1)\left(g_{j}-1\right)+\beta \delta_{j} & \text { if } j \neq \ell \\ (2 \beta-1)\left(g_{\ell}-g\right)+\beta \delta_{\ell} & \text { if } j=\ell\end{cases}
$$

From (3) we get $e_{1}^{(\ell)}+\cdots+e_{t}^{(\ell)}=\delta$ for every $\ell$.

Assume from now on that $\delta_{i, j} \neq 0$ for all $i$ and $j$ if $\beta=1$; otherwise, assume $g_{i} \geq 2$ for every $i$.

Let $\Gamma$ denote the dual graph of $C$, with vertices ordered according to the ordering $C_{1}, \ldots, C_{t}$ of the components of $C$. Recall the notation in Section 5 .

For each $\ell=1, \ldots, t$, let $\underline{n}^{(\ell)}=\left(n_{1}^{(\ell)}, \ldots, n_{t}^{(\ell)}\right)$ be the unique $t$-tuple of integers $\underline{n}^{(\ell)}\left(\underline{e}^{(\ell)}\right)$ claimed by Lemma 4 .

Proposition 5. Recall the setup and notation of Section 2. Let $\ell \in\{1, \ldots, t\}$. Assume that the components of $C$ intersect at points in $\underline{n}^{(\ell)}$-general position. Let $f: \mathcal{X} \rightarrow S$ be a smoothing of $C$ along the general direction. For $\beta=1$, assume $\delta_{i, j} \neq 0$ for all $i$ and $j$; otherwise assume $g_{i} \geq 2$ for every $i$. Then

1. $h^{0}\left(C_{\{\ell\}^{c}}, L_{\frac{n^{(\ell)}}{f \ell\}^{c}}}\left(-\Delta_{\ell}\right)\right)=0$,

2. $h^{0}\left(C_{\{i\}^{c}}, L_{f}^{\underline{n}^{(\ell)},\{i\}^{c}}\left(-\Delta_{i}\right)\right)<\kappa_{\beta}$ for each $i \in\{1, \ldots, t\}-\{\ell\}$,

3. $h^{0}\left(C, L_{\frac{n^{(\ell)}}{f}}\right)=\kappa_{\beta}$.

Proof. For simplicity, assume $\ell=1$. Put $\underline{e}:=\underline{e}^{(\ell)}$ and $\underline{n}:=\underline{n}^{(\ell)}$. Also, let $L \underline{n}:=L \frac{n}{f}$ and $L \frac{n}{J}:=L_{f, J}^{n}$ for each nonempty $J \subseteq\{1, \ldots, t\}$.

We shall prove the first two statements simultaneously. Let $u \in\{1, \ldots, t\}$. Let $I \subseteq\{u\}^{c}$ be a minimal subset such that

$$
H^{0}\left(C_{I}, L_{I}^{n}\left(-\Delta_{I, I^{c}}\right)\right)=H^{0}\left(C_{\{u\}^{c}}, L_{\{u\}^{c}}\left(-\Delta_{u}\right)\right) .
$$

We need only show that $h^{0}\left(C_{I}, L \frac{n}{I}\left(-\Delta_{I, I^{c}}\right)\right)=0$ if $1 \notin I$, and $h^{0}\left(C_{I}, L \frac{n}{I}\left(-\Delta_{I, I^{c}}\right)\right)<$ $\kappa_{\beta}$ if $1 \in I$.

The case $I=\emptyset$ is clear. Suppose $I$ is nonempty. By the minimality of $I$, the restriction map

$$
H^{0}\left(C_{I}, L_{\frac{n}{I}}\left(-\Delta_{I, I^{c}}\right)\right) \rightarrow H^{0}\left(C_{i}, L_{i}^{\underline{n}}\left(-\Delta_{i, I^{c}}\right)\right)
$$

is nonzero for each $i \in I$. So, Lemma 3 yields

$$
\begin{cases}h^{1}\left(C_{I}, L \frac{n}{I}\left(-\Delta_{I, I^{c}}\right)\right)=0 & \text { for } \beta>1 \\ h^{1}\left(C_{I}, L \frac{n}{I}\left(-\Delta_{I, I^{c}}\right)\right) \leq \gamma \frac{n}{I}+\underline{h}^{I} & \text { for } \beta=1 .\end{cases}
$$


Let $\rho:=(2 \beta-1)(g-1)$ if $1 \in I$, and $\rho:=0$ otherwise. Let $\chi_{I}$ denote the Euler characteristic of $\mathcal{O}_{C_{I}}$. Recall the notation in Section 5. We have

$$
\begin{aligned}
\operatorname{deg} L \frac{n}{I} & =\sum_{i \in I} \operatorname{deg} L_{i}^{\underline{n}} \\
& =\sum_{i \in I}\left(2 \beta\left(g_{i}-1\right)+\beta \delta_{i}+\sum_{j \neq i}\left(n_{j}-n_{i}\right) \delta_{i, j}\right) \\
& =\sum_{i \in I}\left(e_{i}+\sum_{j \neq i}\left(n_{j}-n_{i}\right) \delta_{i, j}\right)+\rho+\sum_{i \in I}\left(g_{i}-1\right) \\
& =\sum_{i \in I} \epsilon_{i}^{\underline{e}, \underline{n}}+\rho-\chi_{I}-\sum_{i \in I} \sum_{j \in I-\{i\}} \delta_{i, j} \\
& =\epsilon_{I}^{\underline{e}, \underline{n}}+\rho-\chi_{I} .
\end{aligned}
$$

Thus, by the Riemann-Roch theorem,

$$
h^{0}\left(C_{I}, L \frac{\underline{n}}{I}\left(-\Delta_{I, I^{c}}\right)\right) \leq \epsilon_{I}^{\underline{e}, \underline{n}}-\delta_{I, I^{c}}+\rho+\gamma_{I}^{\underline{n}}+\underline{h}^{I}
$$

for $\beta=1$, whereas

$$
h^{0}\left(C_{I}, L \frac{\underline{n}}{I}\left(-\Delta_{I, I^{c}}\right)\right)=\epsilon_{I}^{\underline{e}, \underline{n}}-\delta_{I, I^{c}}+\rho
$$

for $\beta>1$. So, by Lemma 4 ,

$$
h^{0}\left(C_{I}, L \frac{n}{I}\left(-\Delta_{I, I^{c}}\right)\right) \leq \rho
$$

for $\beta=1$, while

$$
h^{0}\left(C_{I}, L \frac{\underline{n}}{I}\left(-\Delta_{I, I^{c}}\right)\right) \leq \rho-\gamma_{I}^{\beta, 1, \underline{n}+\underline{h}^{I}}
$$

for $\beta>1$. Thus $h^{0}\left(C_{I}, L_{I}^{\frac{n}{I}}\left(-\Delta_{I, I^{c}}\right)\right)=0$ if $1 \notin I$, whereas $h^{0}\left(C_{I}, L \frac{n}{I}\left(-\Delta_{I, I^{c}}\right)\right)<\kappa_{\beta}$ if $1 \in I$, as we wished to show.

As for the third statement, by semicontinuity, $h^{0}\left(C, L^{\underline{n}}\right) \geq \kappa_{\beta}$. So it is enough to show that $h^{0}\left(C, L^{\underline{n}}\right) \leq \kappa_{\beta}$. Now, since $h^{0}\left(C, L^{\underline{n}}\right) \geq \kappa_{\beta}>0$, the first and second statements imply that the restriction map $H^{0}\left(C, L^{\underline{n}}\right) \rightarrow H^{0}\left(C_{i}, L_{i}^{\underline{n}}\right)$ is nonzero for each $i=1, \ldots, t$. Thus, by Lemma 3 , $h^{1}\left(C, L^{\underline{n}}\right) \leq 1$ for $\beta=1$, and $h^{1}\left(C, L^{\underline{n}}\right)=0$ for $\beta>1$. From the Riemann-Roch theorem we get $h^{0}\left(C, L^{\underline{n}}\right) \leq \kappa_{\beta}$, as wished.

Example. We will see that it is necessary to assume $f$ is a smoothing along the general direction in Proposition 5 .

Let $C$ be a projective nodal curve with three irreducible components $C_{1}, C_{2}, C_{3}$, all of them nonsingular. Assume that $C_{1}$ intersects $C_{2}$ and $C_{3}$, each at only one point, denoted by $P_{1,2}$ and $P_{1,3}$, respectively. Assume $C_{2}$ intersects $C_{3}$ at two points, denoted by $P_{2}$ and $P_{3}$. Assume $C_{2}$ has genus 5 , and $C_{3}$ has genus 9 .

Let $\beta:=1$. Then $\underline{e}^{(1)}=(-14,7,11)$ and $\underline{n}^{(1)}=(0,7,8)$, as it can be checked directly. Assume that the points $P_{1,2}, P_{1,3}, P_{2}, P_{3}$ are in $\underline{n}^{(1)}$-general position.

Let $f: \mathcal{X} \rightarrow S$ be a regular smoothing of $C$. Put

$$
M:=L_{f,\{2,3\}}^{n^{(1)}}\left(-P_{1,2}-P_{1,3}\right) .
$$

If the smoothing $f$ is taken along the general direction, Proposition [5 says that $h^{0}\left(C_{\{2,3\}}, M\right)=0$. We claim that, if $f$ is taken along a special direction, then $h^{0}\left(C_{\{2,3\}}, M\right)=1$.

Indeed, let

$$
M_{2}:=K_{2}\left(-7 P_{1,2}+2\left(P_{2}+P_{3}\right)\right) \quad \text { and } \quad M_{3}:=K_{3}\left(-8 P_{1,3}\right) .
$$


Then $\left.M\right|_{C_{2}} \cong M_{2}$ and $\left.M\right|_{C_{3}} \cong M_{3}$. Furthermore, as $f$ ranges through all regular smoothings of $C$, the sheaf $M$ ranges through all invertible sheaves on $C_{\{2,3\}}$ restricting to $M_{2}$ on $C_{2}$ and $M_{3}$ on $C_{3}$. (This fact follows from [6], Cor. 6.9, p. 297 and Lemma 2, as in the proof of Lemma 3.) These sheaves depend on an element $c \in k^{*}$ as explained below.

Fix isomorphisms $\varphi_{i, j}:\left.M_{i}\right|_{P_{j}} \rightarrow k$ for all $i, j \in\{2,3\}$. Then, for each $c \in k^{*}$ there are an invertible sheaf $N_{c}$ on $C_{\{2,3\}}$ and isomorphisms $\alpha_{i}:\left.N_{c}\right|_{C_{i}} \rightarrow M_{i}$ for $i=2,3$ such that $\left.\varphi_{2,2} \alpha_{2}\right|_{P_{2}}=\left.\varphi_{3,2} \alpha_{3}\right|_{P_{2}}$ and $\left.\varphi_{2,3} \alpha_{2}\right|_{P_{3}}=\left.c \varphi_{3,3} \alpha_{3}\right|_{P_{3}}$. Furthermore, given any invertible sheaf $N$ on $C_{\{2,3\}}$ and isomorphisms $\beta_{i}:\left.N\right|_{C_{i}} \rightarrow M_{i}$ for $i=2,3$, there are a unique $c \in k^{*}$ and a unique isomorphism $\gamma: N \rightarrow N_{c}$ such that $\beta_{i}=\left.\alpha_{i} \gamma\right|_{C_{i}}$ for $i=2,3$.

The sheaf $N_{c}$ is simply the kernel of the map

$$
\left(\mu_{2}, \mu_{3}\right): M_{2} \oplus M_{3} \rightarrow k \oplus k,
$$

where, for local sections $s_{2}$ and $s_{3}$ of $M_{2}$ and $M_{3}$,

$$
\begin{aligned}
& \mu_{2}\left(s_{2}, s_{3}\right)=\varphi_{2,2}\left(s_{2}\left(P_{2}\right)\right)-\varphi_{3,2}\left(s_{3}\left(P_{2}\right)\right), \\
& \mu_{3}\left(s_{2}, s_{3}\right)=\varphi_{2,3}\left(s_{2}\left(P_{3}\right)\right)-c \varphi_{3,3}\left(s_{3}\left(P_{3}\right)\right) .
\end{aligned}
$$

Since the points $P_{1,2}, P_{1,3}, P_{2}, P_{3}$ are in $\underline{n}^{(1)}$-general position, $h^{0}\left(C_{i}, M_{i}\right)=1$ for each $i=2,3$, and a generator $\bar{s}_{i}$ of $H^{0}\left(C_{i}, \bar{M}_{i}\right)$ is nonzero at both $P_{2}$ and $P_{3}$. Thus $h^{0}\left(C_{\{2,3\}}, N_{c}\right) \leq 1$ for each $c \in k^{*}$.

Fix generators $\bar{s}_{2}$ and $\bar{s}_{3}$ of the one-dimensional vector spaces $H^{0}\left(C_{2}, M_{2}\right)$ and $H^{0}\left(C_{3}, M_{3}\right)$. In order that $h^{0}\left(C_{\{2,3\}}, N_{c}\right)=1$ it is necessary and sufficient that there be $a, b \in k^{*}$ such that $\mu_{i}\left(a \bar{s}_{2}, b \bar{s}_{3}\right)=0$ for $i=2,3$. Equivalently, the following linear conditions must be satisfied:

$$
\begin{aligned}
a \varphi_{2,2}\left(\bar{s}_{2}\left(P_{2}\right)\right)-b \varphi_{3,2}\left(\bar{s}_{3}\left(P_{2}\right)\right) & =0, \\
a \varphi_{2,3}\left(\bar{s}_{2}\left(P_{3}\right)\right)-b c \varphi_{3,3}\left(\bar{s}_{3}\left(P_{3}\right)\right) & =0 .
\end{aligned}
$$

These are satisfied, for $a, b \in k^{*}$, as long as

$$
c=\frac{\varphi_{3,2}\left(\bar{s}_{3}\left(P_{2}\right)\right) \varphi_{2,3}\left(\bar{s}_{2}\left(P_{3}\right)\right)}{\varphi_{3,3}\left(\bar{s}_{3}\left(P_{3}\right)\right) \varphi_{2,2}\left(\bar{s}_{2}\left(P_{2}\right)\right)} .
$$

Thus, for a smoothing $f$ such that $M \cong N_{c}$ for $c$ as above, $h^{0}\left(C_{\{2,3\}}, M\right)=1$. Our claim is proved, and the example finished.

Let $f: \mathcal{X} \rightarrow S$ be a regular smoothing of $C$. For any invertible extension $\mathcal{L}$ of $\mathcal{K}_{f}^{\beta}(\eta)$ to $\mathcal{X}$, put $V_{\mathcal{L}}:=H^{0}(\mathcal{X}, \mathcal{L})$, and let $\bar{V}_{\mathcal{L}} \subseteq H^{0}(C, \mathcal{L}(s))$ be the subspace generated by $V_{\mathcal{L}}$ under restriction to the special fiber. We say that $\left(\bar{V}_{\mathcal{L}}, \mathcal{L}(s)\right)$ is a limit $\beta$-canonical system.

Let $\mathcal{W}$ be the relative Cartier divisor on $\mathcal{X}$ over $S$ whose generic fibre $\mathcal{W}(\eta)$ is the Weierstrass (Cartier) divisor of the complete $\beta$-canonical system, that is, the complete system of sections of $\mathcal{K}_{f}^{\beta}(\eta)$. Let $W_{f}:=\mathcal{W}(s)$. We call $W_{f}$ the limit Weierstrass divisor of order $\beta$.

To simplify the notation, let $V_{f}^{\frac{n}{f}}:=\bar{V}_{\mathcal{L} \frac{n}{f}}$, viewed inside $H^{0}\left(C, L \frac{n}{f}\right)$.

Theorem 6. Recall the setup and notation of Section 2. For each $\ell=1, \ldots, t$, let $\underline{e}^{(\ell)}$ be the $t$-uple of integers given by (26) , and let $\underline{n}^{(\ell)}=\left(n_{1}^{(\ell)}, \ldots, n_{t}^{(\ell)}\right)$ be the unique $t$-tuple of integers $\underline{n}^{(\ell)}\left(\underline{e}^{(\ell)}\right)$ claimed by Lemma 4 . Assume that the components of $C$ intersect at points in $\underline{n}^{(\ell)}$-general position, for each $\ell=1, \ldots, t$. 
Assume that $\delta_{i, j} \neq 0$ for all $i$ and $j$ if $\beta=1$, and that $g_{i} \geq 2$ for every $i$ if $\beta>1$. Then the following statements hold.

1. Let $f: \mathcal{X} \rightarrow S$ be a smoothing of $C$ along the general direction. For each $\ell=1, \ldots, t$, let $W_{\ell}$ denote the ramification divisor on $C_{\ell}$ of the linear system of sections of $L_{f, \ell}^{n^{(\ell)}}$ generated by $H^{0}\left(C, L_{\frac{n^{(\ell)}}{}}\right)$. Then, as a Weil divisor, $W_{f}=W$, where

$$
W=\sum_{\ell=1}^{t} W_{\ell}+\sum_{i<j} \kappa_{\beta}\left(\kappa_{\beta}-1-n_{j}^{(i)}-n_{i}^{(j)}\right) \Delta_{i, j} .
$$

2. Conversely, let $N_{1}, \ldots, N_{t}$ be a general collection of invertible sheaves on $C$ satisfying

(a) $N_{1} \otimes \cdots \otimes N_{t} \cong \mathcal{O}_{C}$,

(b) $\left.N_{i}\right|_{C_{\{i\}^{c}}} \cong \mathcal{O}_{C_{\{i\}^{c}}}\left(\Delta_{i}\right)$ for each $i=1, \ldots, t$.

For each $\ell=1, \ldots, t$, let $W_{\ell}$ be the ramification divisor on $C_{\ell}$ of the linear system of sections of $\left.L^{(\ell)}\right|_{C_{\ell}}$ generated by $H^{0}\left(C, L^{(\ell)}\right)$, for

$$
L^{(\ell)}:=K^{\beta} \otimes N_{1}^{\otimes n_{1}^{(\ell)}} \otimes \cdots \otimes N_{t}^{\otimes n_{t}^{(\ell)}} .
$$

Then $W$, as given above, is the fundamental cycle of a limit Weierstrass divisor of order $\beta$.

Proof. Let $\ell \in\{1, \ldots, t\}$. From Statement 3 of Proposition 5 we get

$$
V_{f}^{\underline{n}^{(\ell)}}=H^{0}\left(C, L_{f}^{\underline{n}^{(\ell)}}\right) .
$$

In addition, from Statements 1 and 2 we get that, for each $j=1, \ldots, t$, the restriction map

$$
V_{f}^{\underline{n}^{(\ell)}} \rightarrow H^{0}\left(C_{j}, L{\frac{\underline{n}_{f, j}^{(\ell)}}{}}^{(\ell)}\right.
$$

is injective for $j=\ell$ and nonzero otherwise. Thus $\left(V_{f}^{n^{(\ell)}}, L \frac{n_{f}^{(\ell)}}{f}\right)$ is what is called in 4 , p. 26, the limit $\beta$-canonical system associated to $C_{\ell}$. Then the equality $W_{f}=W$ follows from [4, Thm. 7, p. 30. The first statement is proved.

Conversely, it follows from [7, Prop. 3.16 or [6, Thm. 6.10, p. 297 that there is a regular smoothing $f: \mathcal{X} \rightarrow S$ of $C$ such that $\left.N_{i} \cong \mathcal{O}_{\mathcal{X}}\left(C_{i}\right)\right|_{C}$ for each $i=1, \ldots, t$. The enriched structure depends only on the tangent direction at the origin of the arc corresponding to $f$ in the versal deformation space of $C$; see [7, Thm. 3.6. Since the collection $N_{1}, \ldots, N_{t}$ is general, $f$ is a smoothing of $C$ along a general direction. The second statement now follows from the first.

Example. We will see that it is necessary to assume that $\delta_{i, j} \neq 0$ for all $i$ and $j$ if $\beta=1$ in the statement of Theorem [6.

Let $C$ be a projective nodal curve of arithmetic genus $g$ over an algebraically closed field $k$ of characteristic zero. Assume $C$ has three components, $C_{1}, C_{2}$ and $C_{3}$, all of them nonsingular with genera $g_{1}, g_{2}$ and $g_{3}$ greater than 2 . Assume $C_{1}$ intersects $C_{i}$ at a single point $p_{i}$, and that $p_{i}$ is not a Weierstrass point for $C_{i}$, for each $i=2,3$. Assume $C_{2}$ and $C_{3}$ do not intersect. Then $C$ is a curve of compact type.

Let $f: \mathcal{X} \rightarrow S$ be a regular smoothing of $C$. Let $\mathcal{K}_{f}$ be the relative dualizing sheaf of $f$. Let $n_{2}$ and $n_{3}$ be integers, and set $L:=\left.\mathcal{K}_{f}\left(n_{2} C_{2}+n_{3} C_{3}\right)\right|_{C}$. We claim 
that the complete linear system, $H^{0}(C, L)$, of sections of $L$ either has infinitely many base points, or is degenerate on $C_{1}$, or has dimension $h^{0}(C, L)$ greater than g. Thus, if $V \subseteq H^{0}(C, L)$ is the subspace generated by restricting the global sections of $\mathcal{K}_{f}\left(n_{2} C_{2}+n_{3} C_{3}\right)$ to $C$, then either $V \neq H^{0}(C, L)$, or the restriction map $H^{0}(C, L) \rightarrow H^{0}\left(C_{i},\left.L\right|_{C_{i}}\right)$ is zero for a certain $i \neq 1$ or not injective for $i=1$.

Indeed, let $K_{i}$ be the canonical sheaf of $C_{i}$ for $i=1,2,3$. If $H^{0}(C, L)$ has finitely many base points, then $h^{0}\left(C_{i},\left.L\right|_{C_{i}}\right)>0$ for $i=2,3$. So $h^{0}\left(C_{i}, K_{i}\left(\left(1-n_{i}\right) p_{i}\right)\right)>0$, and hence, since $p_{i}$ is not a Weierstrass point of $C_{i}$,

$$
n_{i} \leq g_{i} \text { for } i=2,3 \text {. }
$$

In addition, since $h^{0}(C, L) \geq g$ by semicontinuity, if $H^{0}(C, L)$ is nondegenerate on $C_{1}$, then $h^{0}\left(C_{1},\left.L\right|_{C_{1}}\right) \geq g$. Now,

$$
h^{0}\left(C_{1},\left.L\right|_{C_{1}}\right)=h^{0}\left(C_{1}, K_{1}\left(\left(1+n_{2}\right) p_{2}+\left(1+n_{3}\right) p_{3}\right)\right) \leq g_{1}+n_{2}+n_{3}+2 .
$$

Thus, since $g=g_{1}+g_{2}+g_{3}$, for $h^{0}\left(C_{1},\left.L\right|_{C_{1}}\right) \geq g$ we need that

$$
n_{2}+n_{3} \geq g_{2}+g_{3}-2 .
$$

Combining (27) and (28), we get that $n_{i} \geq g_{i}-2$ for each $i=2,3$. In particular, since $g_{i}>2$ for each $i$, we get that $n_{2}$ and $n_{3}$ are positive.

Now, consider the natural exact sequence,

$$
0 \rightarrow K_{1}\left(n_{2} p_{2}+n_{3} p_{3}\right) \rightarrow L \rightarrow K_{2}\left(\left(1-n_{2}\right) p_{2}\right) \oplus K_{3}\left(\left(1-n_{3}\right) p_{3}\right) \rightarrow 0 .
$$

Since $n_{2}$ and $n_{3}$ are positive,

$$
h^{1}\left(C_{1}, K_{1}\left(n_{2} p_{2}+n_{3} p_{3}\right)\right)=0 \text { and } h^{0}\left(C_{1}, K_{1}\left(n_{2} p_{2}+n_{3} p_{3}\right)\right)=g_{1}+n_{2}+n_{3}-1 \text {. }
$$

In addition, since $p_{i}$ is not a Weierstrass point of $C_{i}$,

$$
h^{0}\left(C_{i}, K_{i}\left(\left(1-n_{i}\right) p_{i}\right)\right)=g_{i}+1-n_{i} \text { for } i=2,3 .
$$

Thus, from the natural long exact sequence in cohomology,

$$
h^{0}(C, L)=\left(g_{1}+n_{2}+n_{3}-1\right)+\left(g_{2}+1-n_{2}\right)+\left(g_{3}+1-n_{3}\right)=g+1 .
$$

\section{REFERENCES}

[1] F. Catanese, Pluricanonical-Gorenstein-curves, In Enumerative geometry and classical algebraic geometry (Nice, 1981), pp. 51-95, Progr. Math., 24, Birkhäuser, Boston, 1982. MR685764 (85e:14041)

[2] D. Eisenbud and J. Harris, Limit linear series: basic theory, Invent. Math. 85 (1986), 337-371. MR846932 (87k:14024)

[3] D. Eisenbud and J. Harris, Existence, decomposition and limits of certain Weierstrass points, Invent. Math. 87 (1987), 495-515. MR874034(88a:14028b)

[4] E. Esteves, Linear systems and ramification points on reducible nodal curves, In Algebra Meeting (Rio de Janeiro, 1996), pp. 21-35, Mat. Contemp., 14, Soc. Bras. Mat., Rio de Janeiro, 1998. MR1662675 (2000b:14035)

[5] E. Esteves and N. Medeiros, Limits of Weierstrass points in regular smoothings of curves with two components, C. R. Acad. Sci. Paris, Série I Math. 300 (2000), 873-878. MR.1771950 (2001k:14063)

[6] E. Esteves and N. Medeiros, Limit canonical systems on curves with two components, Invent. Math. 149 (2002), 267-338. MR1918674(2003e:14017) 
[7] L. Mainò, Moduli space of enriched stable curves, Ph.D. thesis, Harvard University, 1998.

[8] C. S. Seshadri, Vector bundles on curves, In Linear algebraic groups and their representations (Los Angeles, 1992), pp. 163-200, Contemp. Math., 153, Amer. Math. Soc., Providence, 1993. MR:1247504(95b:14008)

Instituto de Matemática Pura e Aplicada, Estrada Dona Castorina, 110, 22460-320 RIO DE JANEIRO RJ, BRAZIL

E-mail address: esteves@impa.br

Departament of Mathematics, ibilce, Universidade Estadual Paulista (UNESP), Rua Cristóvão Colombo, 2265, 15054-000 SÃo José do Rio Preto SP, Brazil

E-mail address: parham@ibilce.unesp.br 